\title{
Near-infrared diffuse optical tomography
}

\author{
A.H. Hielscher ${ }^{\mathrm{a}, *}$, A.Y. Bluestone ${ }^{\mathrm{a}, \mathrm{b}}$, G.S. Abdoulaev ${ }^{\mathrm{a}}$, A.D. Klose ${ }^{\mathrm{a}}$, J. Lasker ${ }^{\mathrm{a}}$, M. Stewart ${ }^{\mathrm{b}}$, \\ U. Netz ${ }^{\mathrm{c}}$ and J. Beuthan ${ }^{\mathrm{c}}$ \\ ${ }^{a}$ Departments of Biomedical Engineering and Radiology, Columbia University, New York, NY, USA \\ ${ }^{\mathrm{b}}$ Departments of Pathology and Physiology and Pharmacology, SUNY - Downstate Medical Center, Brooklyn, NY, \\ USA \\ ${ }^{\mathrm{c}}$ Institut für Medizinische Physik und Lasermedizin, Freie Universität Berlin, Berlin, Germany
}

\begin{abstract}
Diffuse optical tomography (DOT) is emerging as a viable new biomedical imaging modality. Using near-infrared (NIR) light, this technique probes absorption as well as scattering properties of biological tissues. First commercial instruments are now available that allow users to obtain cross-sectional and volumetric views of various body parts. Currently, the main applications are brain, breast, limb, joint, and fluorescence/bioluminescence imaging. Although the spatial resolution is limited when compared with other imaging modalities, such as magnetic resonance imaging (MRI) or X-ray computerized tomography (CT), DOT provides access to a variety of physiological parameters that otherwise are not accessible, including sub-second imaging of hemodynamics and other fast-changing processes. Furthermore, DOT can be realized in compact, portable instrumentation that allows for bedside monitoring at relatively low cost. In this paper, we present an overview of current state-of-the -art technology, including hardware and image-reconstruction algorithms, and focus on applications in brain and joint imaging. In addition, we present recent results of work on optical tomographic imaging in small animals.
\end{abstract}

Keywords: Tomography, instrumentation, image reconstruction, scattering media, brain and joint imaging

\section{Introduction}

Over the last 10 years, considerable progress has been made toward a novel biomedical imaging modality that uses near-infrared (NIR) light $(700 \mathrm{~nm}<\lambda<$ $900 \mathrm{~nm}$ ) to probe biomedical tissue [1-5]. Besides Diffuse Optical Tomography (DOT), various other names commonly are used, such as Photon Migration Tomography (PMT), Medical Optical Tomography (MOT), or just Optical Tomography (OT), to name a few. This technology is based on delivering low-energy electromagnetic radiation, typically through optical fibers, to one or more locations on the surface of the body part under investigation and measuring transmitted and/or backreflected intensities at distances up to $10 \mathrm{~cm}$.

*Corresponding author. Departments of Biomedical Engineering and Radiology, Columbia University, ET 351 Mudd Building, MC8904, 500 W. 120 Street, New York, NY 10027-6623, USA. Tel.: +1 212854 5080; Fax: +1 212854 8725; E-mail: ahh2004@ columbia.edu.
The propagation of light in biomedical tissue is governed by the spatially varying scattering and absorption properties of the medium, which are described in the framework of scattering and absorption coefficients, $\mu_{s}$ and $\mu_{a}$, given in units of $1 \mathrm{~cm}$. Also commonly used is the reduced or transport scattering coefficient, $\mu_{s}^{\prime}=(1-g) \mu_{s}$, where $g \in[(1,1]$ is a parameter that describes the anisotropic scattering properties of tissues [6]. Differences in the refractive index between intracellular and extracellular fluids and various subcellular components such as mitochondria or nuclei, as well as varying tissue densities, give rise to differences in scattering coefficient and g-factors between different tissues [7-9]. Differences in chromophore content and concentration lead to different absorption coefficients. Based on measurements of transmitted and reflected light intensities on the surface of the medium, a reconstruction of the spatial distribution of the optical properties inside the medium is attempted. Typical values found in biomedical tissues are $5<\mu_{s}^{\prime}<20 \mathrm{~cm}^{-1}$, $0.01<\mu_{a}<1 \mathrm{~cm}^{-1}$, and $0.75<g<0.99$ for NIR wavelengths [6]. 
What makes DOT a very challenging imaging problem is the fact that, besides being absorbed, NIR light also is strongly scattered in biological tissues. This results in two major problems. First, only very small amounts of light are transmitted through various body parts, such as the brain or breast. This places special demands on detector technology. Secondly, standard backprojection algorithms, as employed in X-ray-based computerized tomography (CT), have limited applicability, and more complex image-reconstruction algorithms must be employed. On the other hand, DOT offers several advantages over currently existing imaging modalities. For example, the comparatively high speed of the data acquisition allows sub-second imaging of spatio-temporal changes of physiological processes. In addition, the instrumentation is available at a lower cost, is portable, and uses harmless non-ionizing radiation. Furthermore, various kinds of contrast mechanisms (e.g., oxyhemoglobin $\left[\mathrm{HbO}_{2}\right]$, deoxyhemoglobin $[\mathrm{Hb}]$, blood volume, tissue-scattering) complement already available imaging modalities. In initial clinical trials performed by various groups around the world, DOT has shown great promise for brain-blood-oxygenation monitoring in preterm infants, hematoma detection and location, cognition analysis, breast cancer diagnosis, joint imaging, and, most recently, fluorescenceenhanced molecular imaging.

In this paper, we first review the basic instrument components for the three most commonly used DOT modalities. Following an introduction to current stateof-the-art image-reconstruction methods, we focus on applications in brain and joint imaging and provide examples of recent advances toward small-animal imaging systems.

\section{Methods}

In general, optical imaging systems can be divided into three categories: time-domain (TD) systems, frequency-domain (FD) devices, and steadystate-domain (SSD) instrumentation [10]. Timeresolved systems inject very short light pulses into the tissue. Traveling through the tissue, the light pulses are attenuated and broadened in time, which can be measured with the appropriate detectors. In FD systems, the source strength is sinusoidally modulated, typically between 100 and $1000 \mathrm{MHz}$, which results in the propagation of so-called photon-density waves inside the tissue. Data acquisition consists of measuring amplitude differences and phase shifts between the incident wave and the detected wave. SSD systems employ light sources that constantly deliver the same amount of energy to the tissue, and the time-independent intensities are measured at the detectors. Each of these modalities requires different hardware and poses different challenges to the image-reconstruction process. In the following section, we describe the major hardware components for each of these modalities, present an overview of the basic structure of image-reconstruction codes, and discuss various advantages and disadvantages of these three optical imaging methods.

\subsection{Instrumentation}

\subsubsection{Time-domain systems}

In the time-resolved methods, a short laser pulse is used as a probe signal. The light transmitted through the body is recorded as a function of time. The transmitted pulse typically appears broadened and, compared to the input pulse, has a lower peak intensity (Fig. 1a). The time at which the maximum response function is reached is indicative of the mean pathlength of the photons. The higher the scattering coefficient, the longer the mean pathlength and the later the maximum is reached. The decreasing slope of the response curve yields information about the absorption coefficient. The higher the absorption, the steeper the slope [11].

The most commonly used technique to obtain timeresolved data for DOT is time-correlated single-photon counting [12-15]. Figure 1b shows the basic components of this type of measurement. The usual light sources are laser diodes, which are driven by a picosecond light pulser. Light pulses with a duration of 10-50 ps (FWHM = full width at half maximum) are emitted at a repetition rate of $1-50 \mathrm{MHz}$. The peak power reaches no more than approximately $100 \mathrm{~mW}$. Typically, wavelengths between $780 \mathrm{~nm}$ and $830 \mathrm{~nm}$ are used. The light is guided through optical fibers to various positions on the tissue. Optical fibers collect the re-emitted photons and guide them to a microchannel plate photomultiplier (MCP-PMT). The MCP-PMT signals are input to a constant fraction discriminator (CFD) via an amplifier and an attenuator. The CFD output is fed to a time-to-amplitude converter (TAC) as a "start counting" signal. The TAC outputs are counted as discrete events by a pulse-height analyzer (PHA) and accumulated until the peak count reaches 100,0001,000,000 counts. The time-response curves obtained then are stored into a personal computer. By placing the detector and source fiber directly against one an- 

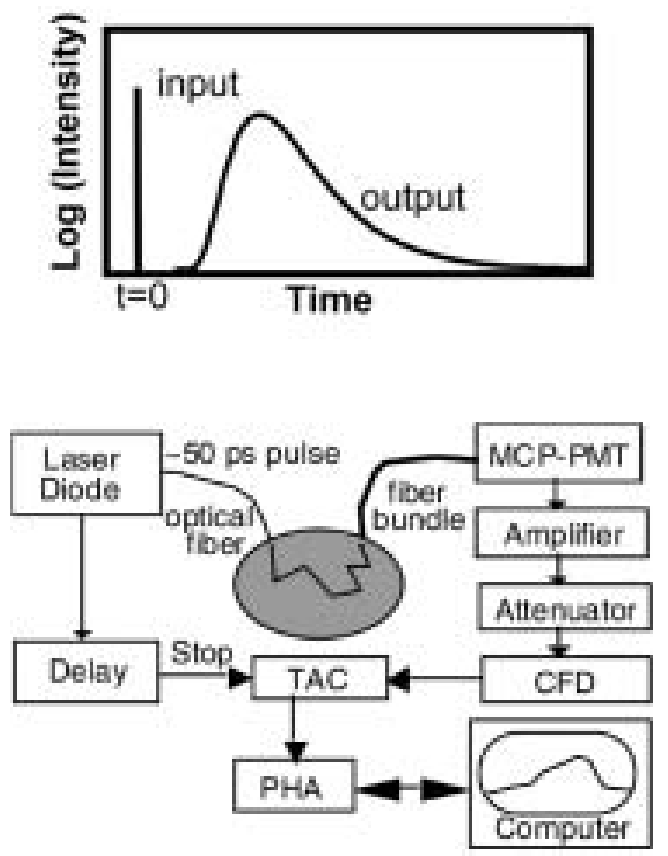

Fig. 1. (a-top): Time-resolved impulse response of highly scattering biological tissue. $\mathrm{A} \sim 50 \mathrm{ps}$ input pulse typically is broadened to 1-10 ns after traveling through 5-10 cm tissue. (b-bottom): Experimental set-up for time-correlated photon counting. (MCP-PMT $=$ Micro-channel-plate Photomultiplier, CFD $=$ Constant Fraction Discriminator, TAC $=$ Time to Amplitude Converter, PHA $=$ Pulse Height Analyzer).

other, the instrument function is measured either before or after the measurements. The instrument function is used to deconvolute the results from the measurements to yield the corrected time-dependent response function. More detailed descriptions of the two most advanced time-resolved optical tomography systems are presented by Ntziachristos et al. [14] and Schmidt et al. [15].

\subsubsection{Frequency-domain systems}

Instead of using a short light pulse, FD systems use sinusoidally amplitude-modulated light sources. The modulation frequency typically is between 100$1000 \mathrm{MHz}$ [16-18]. The measured parameters are the phase shift, $\Phi$, and the demodulation, $M=$ $\left(A C_{o} / D C_{o}\right) /\left(A C_{i} / D C_{i}\right)$, of the light transmitted through the tissue relative to the incident light. Here, $A C_{i}$ and $D C_{i}$ refer to AC amplitudes and DC offset of the intensity of the light sent into the medium; and $A C_{o}$ and $D C_{o}$ are the AC amplitude and DC offset measured after this light has traveled through the medium to the detector (Fig. 2a). Measuring $\Phi$ and $\mathrm{M}$ for all frequencies amounts to performing the Fourier transform
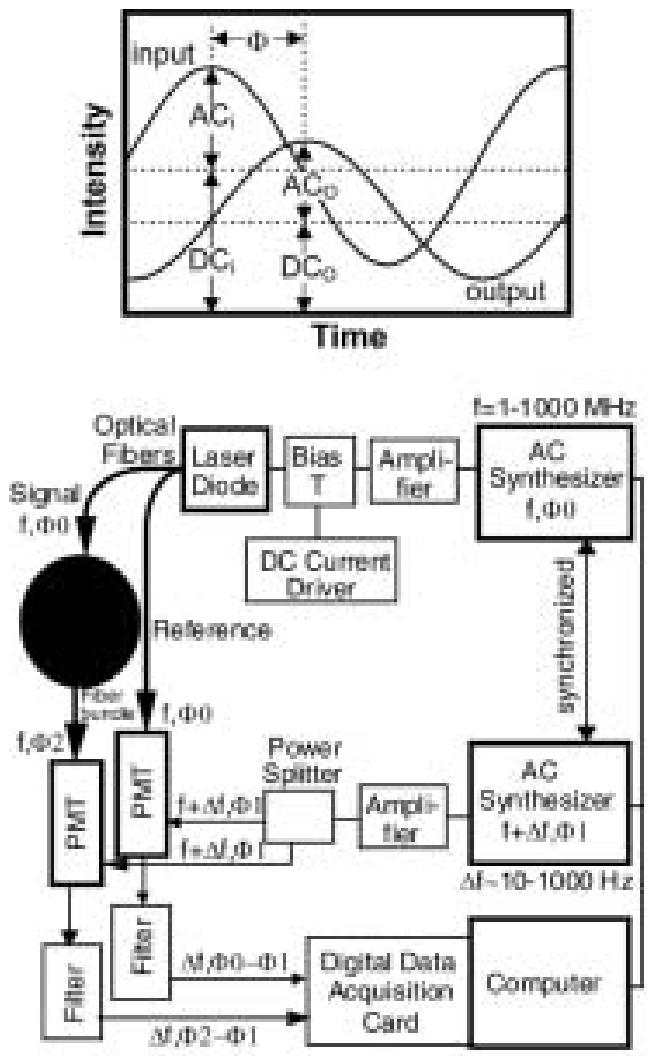

Fig. 2. (a-top): Principle of frequency-domain measurements of light transmitted through biological tissue. (b-bottom): Experimental set-up for cross-correlation measurements of the phase shift $\Phi$ and demodulation M. (PMT = Photomultiplier Tube).

of the TD data. The technical advantage of making measurements in the FD lies in the simpler and more cost-effective hardware design.

An FD device consists basically of four parts: (1) the light source and intensity modulation techniques, (2) the light-delivery system from the diode to the tissue, (3) the light collection and detection, (4) the crosscorrelation technique for measuring the phase shift $\Phi$ and demodulation M (Fig. 2b). Readily available 1$100 \mathrm{~mW}$ laser diodes provide enough light to probe, for example, the brain or breast. To intensity-modulate laser diodes, two components are necessary. First, a DC current is set between the lasing threshold current, $I_{\text {thres }}$, and the maximal allowed current, $I_{\max }$. An AC source provides the amplitude modulation between $I_{\max }$ and $I_{\min }$. Both signals are added together in a Bias-T, which prevents the rf power from entering the DC current source, and visa versa. Optical fibers are used to bring the light from the diode to the tissue and to detect the light transmitted through the medium. Photomultiplier tubes (PMTs), silicon pho- 
todiodes, or avalanche photodiodes usually are used as light detectors [19]. Cross-correlation techniques [2022] transform the cumbersome rf frequencies of the light modulation into signals in the range below $1 \mathrm{kHz}$. Measurements of $\Phi$ and $\mathrm{M}$ are easy to perform at these lower frequencies with accurate digital techniques [23]. One frequency synthesizer with a frequency $\omega=2 \pi f$ is used to intensity-modulate the laser diodes by modulating the drive current of the diode. A second synthesizer with a slightly different frequency, $f+\Delta f(\Delta f \sim 10--1000 \mathrm{~Hz})$ is used to modulate the gain of the PMTs in the reference and signal channel by sinusoidally modulating the voltage of the second dynode. Typically, intensity-modulation frequencies below $1000 \mathrm{MHz}$ are used. In this way, the difference frequency (of between the light-source modulation and the gain modulation is generated within the PMTs. The PMT acts as a mixer. The difference frequency in the $\mathrm{kHz}$ range can be filtered easily from the rf frequencies by a digital data acquisition system [23]. It can be shown that these low cross-correlation frequencies contain the same phase and modulation information as do the rf frequencies [20]. The phase shift can be measured typically within $0.05^{\circ}$ and the demodulation within 0.002 . Currently available FD systems mostly are employed in optical breast imaging [24-27] and, to a somewhat lesser extent, in functional brain imaging [28,29].

\subsubsection{Steady-state-domain systems}

Originally thought to be of limited use, SSD systems have made a comeback in recent years and now are among the most widely used systems in clinical settings. In SSD systems, the light source continuously emits light into the tissue and the transmitted light intensities are measured (Fig. 3a). An example of such a system is the dynamic near-infrared optical tomography (DYNOT) instrument, recently developed by Schmitz et al. [30-32] (Fig. 3b). In this system, two wavelengths $(700 \mathrm{~nm}<\lambda<850 \mathrm{~nm}$ ) are provided by two laser diodes, whose light is coupled sequentially into 32 different fiber bundles that deliver the light to various positions on the surface of the tissue to be studied. Fast switching between different source positions is possible by means of an optical demultiplexer, which consists of a mirror that is rotated by a microprocessor-controlled brushless DC servomotor. A motion-control unit allows up to $\sim 50$ start-stop motions per second. Each source fiber bundle forms one branch of a bifurcated fiber bundle that joins another branch. This second branch is used for light detection.
Each detector fiber bundle terminates on a single silicon photodiode of a multi-channel detection module. This module incorporates analog signal conditioning hardware, such as adjustable gain stages to increase the dynamic range of detection, four lock-in amplifiers, and sample-and-hold circuits that improve signal quality and are necessary for timing purposes. The output voltages of the detector channels are measured by a data-acquisition board and stored on a personal computer. The optical power sent to the target is about $10 \mathrm{~mW}$. A full tomographic data set (two wavelengths at $32 \times 31=992$ source-detector configuration) can be obtained in approximately 0.5 seconds, leading to a data rate of $2 \times 992 / 0.5=3968$ measurements/second, which is the fastest data-acquisition rate (measurement points/per second) of all currently available multisource-detector NIR optical instruments. Using fewer sources, the acquisition time decreases approximately linearly. Similar instruments have been developed by Siegel et al. [33], Colak et al. [34], and Yamashita et al. [35].

\subsection{Image reconstruction algorithm}

\subsubsection{Backprojection methods}

Although it is known that light is strongly scattered in biological media and does not travel in a straight line between source and detector, some researchers implemented backprojection algorithms for DOT [36-38]. In this case, optical signals measured on the surface of the medium are fitted to analytical expressions based on diffusion theory [16,39] to give effective absorption and transport-scattering coefficients, $\mu_{a}$ and $\mu_{s}^{\prime}$, respectively. Analagous to backprojection algorithms in X-ray tomography [40], these effective values are backprojected either on a straight line between source and detector or on a line of most likely path, which may be curved. This results in a map of spatially dependent optical properties inside the medium under consideration. A generalization of this approach is not to back-project onto a line but onto the entire volume, assuming that a spatially varying probability exists that a photon has passed a certain place within the tissue [41, 42]. Depending on the probability, different weights are assigned to different regions of the tissue.

In recent years, topographic methods that rely on backprojection algorithms have found widespread application, especially in functional imaging. These maps project the cortical response during various protocols together with the superficial vascular changes onto a two-dimensional (2D) surface map. Results usually 

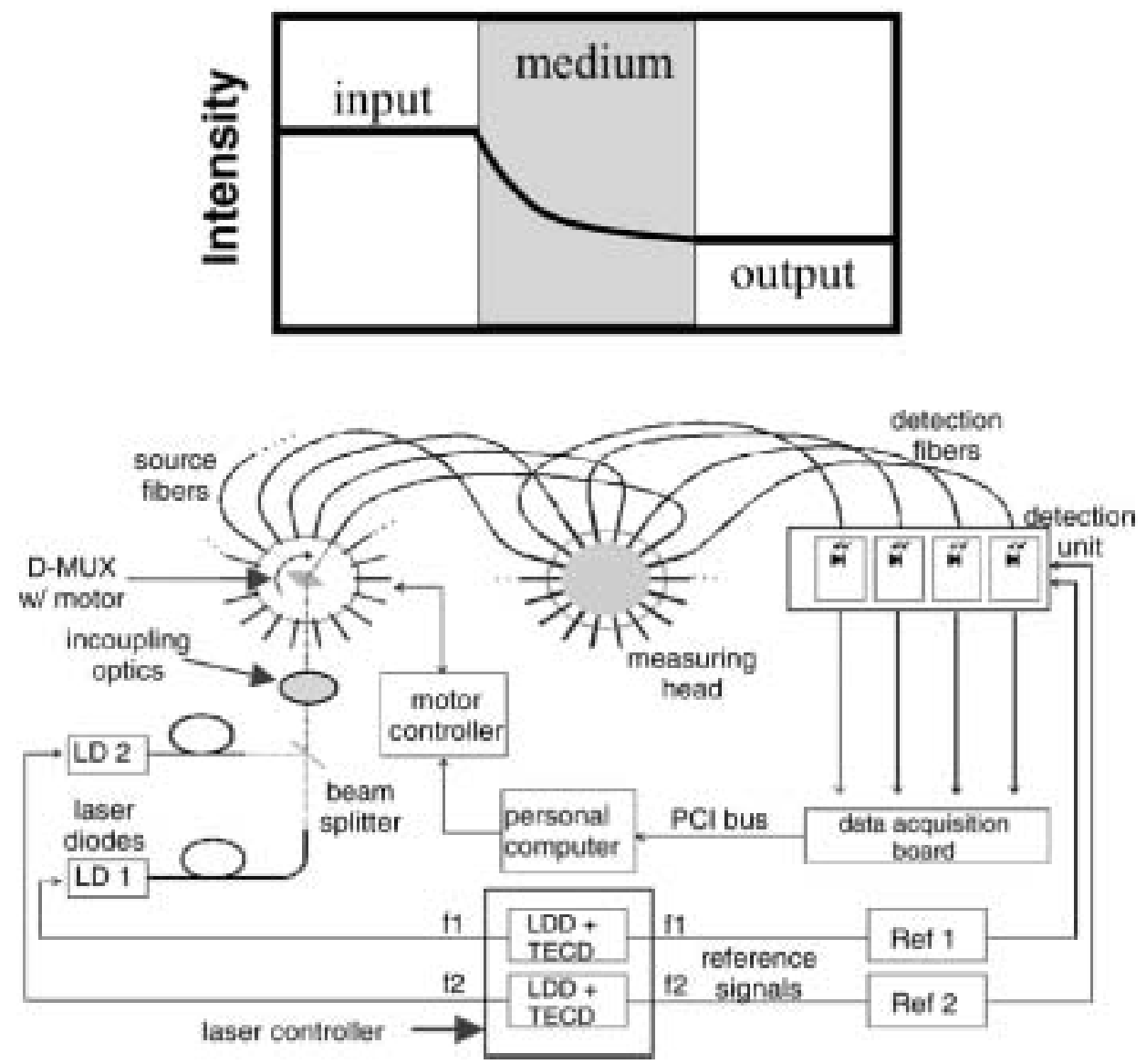

Fig. 3. (a-top): Principle of steady-state domain (SSD) measurements of light transmitted through biological tissue. (b-bottom): Basic block diagram for SSD instrument set-up. LDD = laser diode drivers, TECD $=$ thermo-electric cooling drivers, f 1 and $\mathrm{f} 2=$ diode current modulation frequencies, LD1 and LD2 = laser diodes, D-MUX = optical demultiplexer with servomotor.

are obtained by back-projecting measured light intensities between corresponding source-detector pairs multiplied by the appropriate pathlength factor to obtain the change in the absorption coefficients along the path [29, $43,44]$. All of these approaches yield some results in which positions of inhomogeneities often are recovered quickly, with relatively good precision. However, the absolute values of absorption and scattering generally are not reconstructed with good accuracy and the resolution is lower than it is with iterative methods, which will be discussed next.

\subsubsection{Model-based iterative image reconstruction}

A more accurate reconstruction can be obtained if model-based iterative image reconstruction (MOBIIR) algorithms are applied. Iterative reconstruction schemes are more time consuming, and usually consist of three components (Fig. 4). The first component is a forward model that provides a prediction of the measurements based on a guess of the system parameters (here, the spatial distribution of $\mu_{a}$ and $\mu_{s}^{\prime}$ ). Second, a scheme is used that compares the predicted data with the measured data, which results in some sort of error function, often also called an objective function or norm. The third component is an efficient way of updating the system parameters of the forward model, which in turn provides a new set of predicted data.

Several groups have developed model-based algorithms for optical tomography in recent years [4553]. Codes from different groups differ mainly in what type of forward model they use, what type of updating scheme is employed, and how many iterative updates of the initial guess of optical properties are made. We recently developed MOBIIR algorithms that use as a forward model either finite-difference or finiteelement discretizations of the time-dependent or timeindependent diffusion equation as well as the equation of radiative transfer [54-59]. The results of the forward 


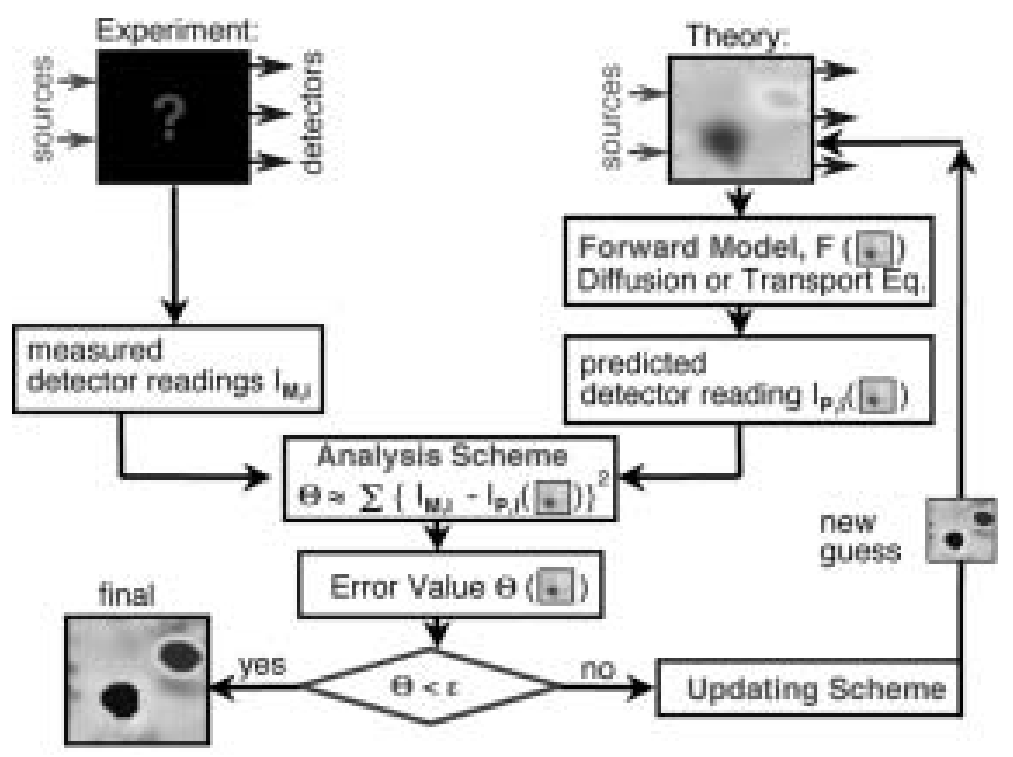

Fig. 4. Basic structure of model-based iterative image reconstruction scheme.

calculations are compared to measurements or to simulated data by using the least square error norm, which can include various assumptions about prior knowledge of the system parameters. The derivatives or gradient of the norm with respect to the system parameters $\left(\mu_{a}(r)\right.$, $\left.\mu_{s}^{\prime}(r)\right)$ are calculated with an adjoined-differentiation method. The advantage of this approach is that the gradient calculation parallels the forward computation in complexity and can be accomplished in a time, of the same order of magnitude as it takes to complete a forward calculation.

\subsection{Comparison of different optical imaging modalities}

The information content as well as complexity of hardware and imaging software increases from SSD to FD and TD systems. The obtainable spatial resolution, given the same number of sources and detectors, is thought to increase from SSD to FD to TD systems. On the other hand, SSD systems allow for much faster data acquisition than do TD systems. While SSD systems allow for data-acquisition rates for one source and one detector of up to $8000 \mathrm{~Hz}$, FD systems currently are limited to $\sim 10-400 \mathrm{~Hz}$, and TD systems typically are limited to less than $1 \mathrm{~Hz}$ [30]. This makes SSD systems the ideal systems to look at fast physiological changes, such as hemodynamic changes in the brain or limbs. Time-resolved systems typically take more than one minute, and rapid changes such as hemodynamic responses to stimuli cannot be imaged.
Another important aspect of DOT is the capability to distinguish between scattering and absorption effects. In a theoretical study, Arridge et al. [60] showed that, if SSD-type measurements are used alone, absorption and scattering effects may be indistinguishable. Therefore, from measurements on the surface alone, the observer will be unable to conclude whether a change in signal is caused by a local change in the absorption coefficient or by a change in the scattering coefficient inside the medium. The image-reconstruction problem is said to be "ill-posed." FD and TD systems contain more information and typically are believed to provide better separation between absorption and scattering effects. However, as Arridge points out, if the refractive index is unknown, which generally is the case, even FD and TD measurements are not enough. However, also as noted by Arridge, "in practice, in inverse problems one solves a regularized problem wherein a combination of likelihood term and a term representing some constraints on the solution is optimized." Under these practical circumstances, separation of absorption and scattering may well be achieved. Barbour et al. [61] and Jiang et al. [62-64] recently presented such practical algorithms for the SSD case. Furthermore, often one can make reasonable assumptions concerning the scattering coefficient and only reconstruct absorption images. For example, it is argued often that scattering changes over the wavelength range considered are small and, hence, can be neglected.

An issue that affects all three optical imaging modalities is the problem of obtaining absolute optical prop- 
erties rather than only changes in optical properties. Typically, absolute values are harder to obtain than are relative changes because absolute attenuation must be measured. This has proven difficult in practice, even though some groups have developed careful calibration schemes and numerical methods and have performed the first quantitative hemoglobin tomography studies [65-67]. These studies, however, have been limited to numerical problems and optical tomographic breast imaging. Most currently available optical devices employed for brain imaging provide only measures of changes or of differences with respect to a reference material or reference physiological state (rest vs. activity). An example is a measurement of optical properties during a breath-hold experiment: The patient is monitored before the breath hold. With the start of the breath hold, the measured optical signals will change, and these changes can then be used to determine changes in oxy- and deoxyhemoglobin. However, because the absolute oxyhemoglobin and dexoyhemonglobin concentrations were not known at the start of the experiments, the absolute concentrations also are not known afterward. Absolute measurements are difficult to obtain because the coupling coefficient between tissue and fibers, as well as losses in optical fibers due to different bending and coupling into the detectors, are difficult to determine. Therefore, the error in absolute measurements $(\sim 1 \%$, see e.g., reference [64]), even when carefully calibrated, typically is larger than the error in relative measurement schemes, where one can determine changes in transmitted light intensities with an accuracy of $\sim 0.01 \%$ (see e.g., reference [68] and Fig. 13a of this manuscript).

Finally, the importance of an accurate forward model that describes light propagation in tissue should be stressed. If the forward model does not accurately describe the propagation of photons inside the medium, the model-based reconstruction scheme will fail. At present, most algorithms rely on the validity of the diffusion approximation as opposed to the generally more accurate equation of radiative transfer $[69,70]$. While in many cases the diffusion theory is indeed a good approximation for describing light propagation in biological tissues, several researchers have determined theoretically and experimentally what the limits of this approximation are [71-77]. For example, it has been shown that the diffusion approximation fails when small-sample geometries are considered in which source-detector separations are small and boundary effects are dominant. Furthermore, radiative-transfer theory is more accurate when the medium contains regions in which the absorption coefficient is not much smaller than is the scattering coefficient or when regions are considered in which the scattering and absorption are very low; so-called "void-like" regions. Turbid media that contain void-like areas play an important role in several biomedical imaging applications. For example, the highly scattering brain tissue is enclosed in a layer of almost clear cerebrospinal fluid, which has very low scattering and absorption coefficients. How this layer affects light propagation recently has been the subject of many studies and discussions [see e.g. [65,69-71].

An example that demonstrates the importance of an accurate forward model is shown in Fig. 5. Figure 5a shows the geometry of a tissue phantom that is made of highly scattering resin $\left(\mu_{a}=0.35 \mathrm{~cm}^{-1}\right.$, $\left.\mu_{s}^{\prime}=11.6 \mathrm{~cm}^{-1}\right)$, which contains a ring of water. Directing laser light onto one side of this sample, we measured the transmitted light intensities at the adjacent side and at the far side of the sample and compared the results with a diffusion-theory code and with a radiative-transfer-theory code. As Figs 5b and 5c show, the experimental results agree well with the numerical results obtained with the radiative-transfer-theory algorithm, and considerable difference exists between the diffusion theory results and experiments. If the experimental results are input to an image-reconstruction code, only the radiative-transfer-based algorithm finds the water ring, while the diffusion code converges to an incorrect result (Fig. 6).

\section{Applications}

\subsection{Brain imaging}

The early application of optical methods for brain imaging concentrated on the detection of hemorrhages and hematomas in the brain. Because hematomas are a localized mass of extravasated blood, and NIR light is strongly absorbed by blood, subcranial hematomas can be detected relatively easily. Since the early 1990s, various groups have reported on the development of instrumentation to detect these bleeds, and the first commercial systems were geared toward this application [78-81]. With the ongoing advances in instrumentation and image-reconstruction algorithms, the focus of DOT in brain imaging has shifted in recent years to two more challenging areas; namely stroke imaging and functional imaging. Next, we provide a review of these areas and present the latest developments in threedimensional (3D) optical tomographic brain imaging. 

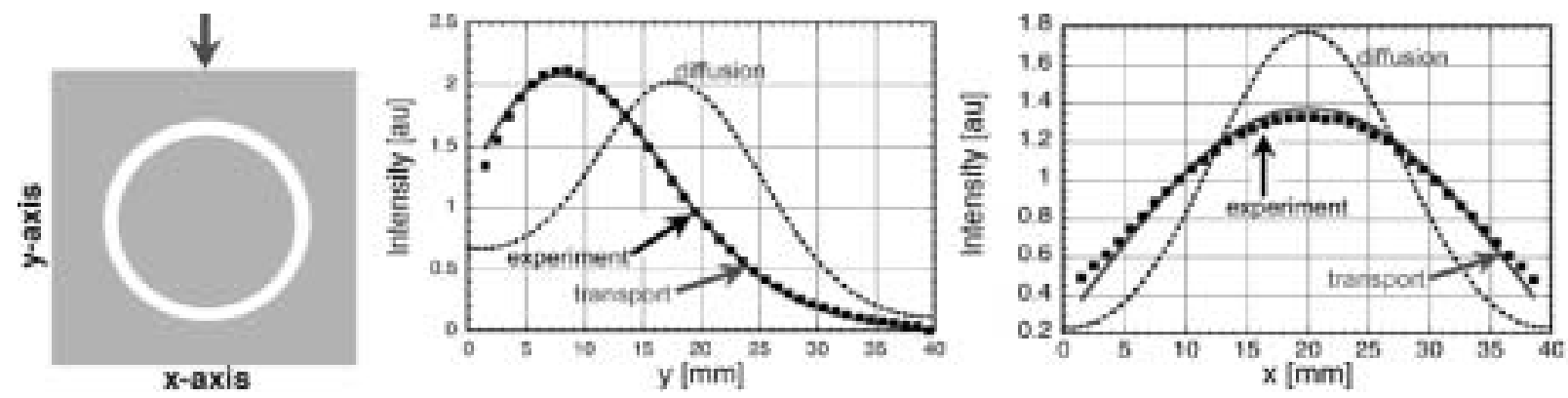

Fig. 5. Experimental and numerical results for a highly scattering medium $\left(\mu_{s}=(1-g) \cdot \mu_{s}=10 \mathrm{~cm}^{-1}\right)$ that contains a low-scattering, water-filled ring. For an incident laser beam (arrow left figure), the transmitted light intensities along the y-axis (middle) and x-axis (right) were measured. In addition, numerical calculations were performed with a 3D finite-difference algorithm that used either the diffusion equation or the equation of radiative transfer. Clearly visible are the good agreement between measurements and the transport-forward model, whereas the diffusion-forward model is less accurate.

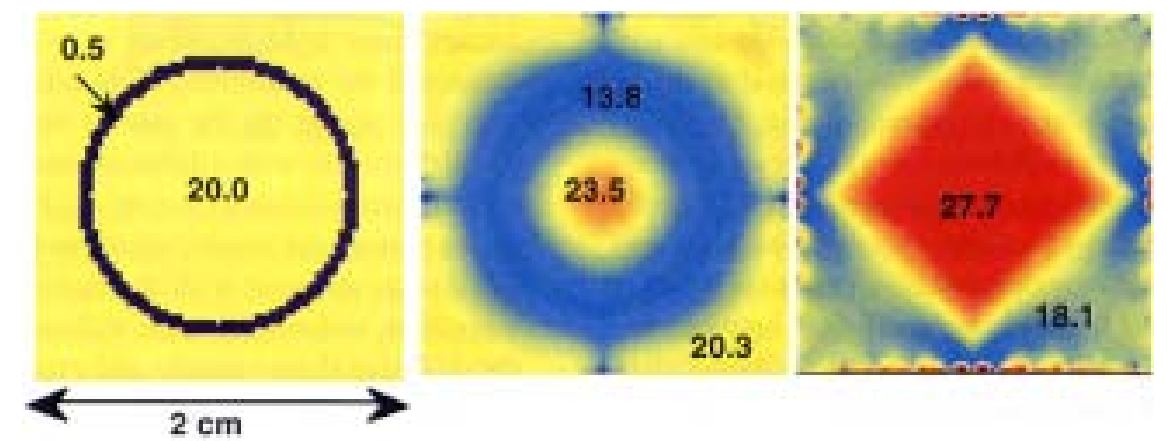

Fig. 6. Reconstruction results illustrating the importance of an accurate forward model. When a transport-theory-based algorithm is used, the ring is recovered (middle). A diffusion-theory-based code does not find the ring and converges to a wrong result (right). (The figure to the left shows the original structure of the medium. Numbers in figures give the reduced scattering coefficient $\mu_{s}^{\prime}$ ).

\subsubsection{Optical functional imaging}

In the last five years, functional brain imaging with near-infrared optical methods has become of great interest [28,29,82-88]. Several groups have reported on changes in the optical signals during various tasks. For example, Benaron et al. [84] studied physiological changes in brain oxygenation in male adults during mixed motor and sensory cortex activation. Hoshi et al. [82] obtained quantitative images of hemoglobin concentration changes associated with neuronal activation in the human brain during a forward (DF) and backward (DB) digit-span task that assesses verbal working memory, and Franceschini et al. [29] reported on imaging arterial pulsation and motor activation in healthy human subjects.

These works are based on multi-wavelength measurements that allow for calculation of changes in optical properties. For example, assuming that the primary influences on the changes in the absorption coefficients at each wavelength are a linear combination of oxyhemoglobin and deoxyhemoglobin, one arrives at [89,
90]:

$$
\Delta \mu_{a}^{\lambda}=\varepsilon_{H b O}^{\lambda} \Delta[H b O]+\varepsilon_{H b}^{\lambda} \Delta[H b O] .
$$

By simultaneously solving a set of algebraic equations at the disparate wavelengths $\left(\lambda_{1}, \lambda_{2}, \ldots, \lambda_{N}\right.$, it is possible to calculate the true concentrations of the $N$ chromophores of interest. For the case of two chromophores consisting of oxyhemoglobin and deoxyhemoglobin, the equations are given by:

$$
\begin{aligned}
\Delta[H b] & =\frac{\varepsilon_{H b O}^{\lambda_{2}} \Delta \mu_{a}^{\lambda_{1}}-\varepsilon_{H b O}^{\lambda_{1}} \Delta \mu_{a}^{\lambda_{2}}}{\varepsilon_{H b}^{\lambda_{1}} \varepsilon_{H b O}^{\lambda^{2}}-\varepsilon_{H b}^{\lambda_{2}} \varepsilon_{H b O}^{\lambda_{1}}} \text { and } \\
\Delta[H b O] & =\frac{\varepsilon_{H b O}^{\lambda_{2}} \Delta \mu_{a}^{\lambda_{1}}-\varepsilon_{H b O}^{\lambda_{1}} \Delta \mu_{a}^{\lambda_{2}}}{\varepsilon_{H b}^{\lambda_{1}} \varepsilon_{H b O}^{\lambda^{2}}-\varepsilon_{H b}^{\lambda_{2}} \varepsilon_{H b O}^{\lambda_{1}}},
\end{aligned}
$$$$
(2 a, b)
$$

where $\lambda_{1,2}$ indicates the wavelength, and $\varepsilon_{H b}$ and $\varepsilon_{H b O}$ are the known extinction coefficients for deoxyhemoglobin and oxyhemoglobin at the given wavelengths, respectively. The $\Delta \mu_{a^{\prime}} s$ in Eqs $2 \mathrm{a}$ and $2 \mathrm{~b}$, at 
each of the two wavelengths, are the calculated changes in the absorption coefficients at each node of the mesh determined using the reconstruction algorithm. Other chromophores that often are included in the analysis are water and cytochrome oxidase. If, in addition to changes in oxy- and deoxyhemoglobin concentrations, water and cytochrome concentrations are changing during a given experiment, four instead of two wavelengths need to be used.

Using this or similar analysis schemes, it has been established clearly that NIR can be used to probe the brain for changes in blood oxygenation and blood volume. Although it is unchallenged that changes in hemodynamic responses to stimuli can be detected, conflicting results have been reported concerning the detectability of scattering changes due to neural activity. The hemodynamic response to various stimuli typically occurs with latencies between 0.5 to 5 seconds. Some groups have reported the detection of faster changes in transmitted optical signals, with latencies of about 50-100 msec. However, these signals are subtler than are the hemodynamic responses, and different groups come to different conclusions. Gratton et al. [91,92] contend that these fast signals are visible only in FD phase-shift measurements, where phase delays of up to 0.3 degree have been observed. Steinbrink et al. [93] recently challenged that contention and reported that they were unable to detect a change in phase shift. Instead, they detected a $0.05 \%$ change in the intensity of the signal, with latencies between 60-160 msec after electrical median nerve stimulation. Several other groups [94, 95] currently are investigating this phenomena and a final conclusion still is outstanding.

\subsubsection{Optical stroke monitoring}

As early as 1993, Hirtz speculated [96] that NIR imaging may be used in the future to "predict stroke or study changes in response to pharmacological agents." NIR optical systems promise several advantages over current brain-imaging techniques such as MRI and CT when applied to critically ill stroke patients [97]. Unlike MRI, CT, or positron emission tomography (PET), optical systems are portable and can be brought to the bedside to monitor critically ill patients. Stroke patients frequently are unstable and unable to tolerate transport to CT or MRI scanner facilities and do not tolerate the repeated scanning necessary to follow an ongoing or evolving condition. In addition, most established imaging techniques require exposure to noxious agents, such as intravenous contrast, radiation, or radioactive emitters. In the critically ill stroke patient, in whom cerebral blood flow, blood volume, and brain oxygenation constantly are changing, a continuous imaging technology is highly desirable.

Initially, researchers sought to monitor cytochrome oxidase during stroke with NIR techniques [98,99]. These investigators used SSD instrumentation with three or form wavelengths and data-analysis algorithms developed since the mid-1970s by Jöbsis et al. [100, 101]. However, the accuracy and validity of these measurements were a point of strong contention. For example Miyake et al. [102] argued “. . . that cytochromes are difficult to detect in blood-containing brains where the path-lengths are too short to measure accurately," while in bloodless brains they "found clear cytochrome $\mathrm{c}$ and aa3 absorption of iron and copper." More recent studies that involved more than 100 wavelengths are more promising as the reported results indicate that, in this case, changes in cytochrome concentration can be decoupled from hemoglobin concentration changes [103, 104].

In 1996, Wolf et al. [105] reported on the use of NIR spectroscopy for non-invasive on-line detection of cortical spreading depression (CSD) in a pento-barbitaltreated rat. Typically, an acute increase of regional cerebral blood flow (rCBF) can be observed during this process [106-108]. Wolf et al. performed studies by measuring transmitted NIR intensities at four wavelengths with an optical probe over the intact skull consisting of one delivery fiber and another detection fiber about $6 \mathrm{~mm}$ apart. One year later, the same group of authors reported on optically measuring peri-infarct depolarization (PID) in focal cerebral ischemia in rats [109]. The authors concluded that NIR imaging is capable of non-invasive detection of a "fingerprint" of PID in rats, which distinguishes it from spreading depression.

In 1999, Vernieri et al. [110] reported on a study that involved the effects of hypercapnia on the near-infrared signal in two patients who had suffered strokes three and four months earlier, with monohemispheric lesions in the middle cerebral artery (MCA) territory. Using a commercial NIR system (OxiPlex by ISS Inc, Champaign, IL) with two light-emitting diodes $(\lambda=825 \mathrm{~nm})$ and two detectors, signal changes were measured during hypercapnia induced by inhalation of a mixture of $7 \% \mathrm{CO}_{2}$ /air for about 90 seconds. In both cases, a decrease (5-10\%) in the backscattered light intensity, corresponding to the expected increase in blood volume, was appreciable only in the unaffected hemisphere. The NIR signal showed no response in the affected hemisphere.

Two more case studies involving two patients who suffered occlusive strokes of the MCA were reported by 
Nemoto et al. [111]. In their studies, the authors used the INVOS 3100A from Somanetics Corp., Troy, MI. This device employs one source and two detectors at different distances to obtain a coarse depth resolution. The cerebral oximeter sensor was placed overnight over the right frontal temporal cortex of a patient with MCA occlusion. While wearing the oximeter sensor, the patient developed a sudden bradycardia that progressed to asystole. The instrument clearly showed the rapid desaturation of the brain after arrest. In the second case, oximetry readings were obtained in a patient with a right internal artery occlusion and an infarct in the MCA territory. Differences in the oximeter readings were obtained for the infarct regions and the border zone between the MCA regions and normally perfused anterior cerebral artery territory.

In a general review paper on non-invasive optical functional imaging of the human brain, Benaron et al. [78] presented a spatially resolved optical tomographic image of a frontal lobe stroke. CT and timeresolved optical imaging were performed sequentially in an infant with hypoxic-schemic injury. The dataacquisition time for the time-resolved optical measurement was six hours. The reconstructed image was generated with a tomographic backprojection algorithm. The authors observed overlap between the optical and CT localization of the injury site and noted that only optical scanning yielded quantitative measurement of regional cortical saturation.

Most recently, Chen et al. [112,113] performed optical studies using an intracranial infarction model in rats. The NIR system used in this study operated in the continuous wave mode and employed one source and one detector separated by $5 \mathrm{~mm}$. The probe was placed sequentially in 20 different positions on the head of the rat, which resulted in a topographic map of optical density changes between pre- and post-MCA. In addition, MRI images were obtained on a Bruker Biospec 2000 with a vertical bore and magnetic field strength of $4.7 \mathrm{~T}$. A T2-weighted MRI was used to identify the location of ischemia and to estimate the lesion area. Furthermore, a triphenyltetrazonlium-chloride stain was used to locate the occlusion focus area. The authors reported "significant" correlation among findings by NIR, MRI, and staining.

These studies clearly demonstrate the potential of optical techniques for stroke imaging. However, most of the work in optical stroke imaging so far provides only topographic maps or uses single-source detector measurements to distinguish between the left and right hemispheres. An important next step is the develop- ment of 3D image-reconstruction schemes that provide sufficient depth resolution to localize the affected areas inside the brain.

\subsubsection{Volumetric brain imaging}

Our group recently went beyond topographic maps and obtained 3D, volumetric reconstructions of the changes in optical properties inside a human head [114]. To illustrate the performance of this approach, we describe a volumetric reconstruction of the vascular reactivity in the brain during a Valsalva maneuver.

The experiment was designed to look at functional hemodynamic changes induced by a Valsalva maneuver in the forehead of a single patient. For the measurement, the patient was placed in the supine position. Three epochs, consisting of Valsalva maneuvers with one-minute rest periods interspersed, were performed. During the Valsalva maneuver, a forced expiration against a closed glottis demonstrates the effects of changes in intrathoracic pressure on blood pressure, and the brain's autoregulatory response to decreased vascular perfusion pressure in cerebral vessels [115117].

Figure 7a shows the locations of the sources and detectors on the forehead. A three-tiered band was used to secure three sets of five optodes per tier to the left forehead. Each optode consisted of a co-located source and detector, and all measurements were performed simultaneously at $760 \mathrm{~nm}$ and $830 \mathrm{~nm}$. Using two wavelengths and the scheme described in Eqs 1 and 2, we calculated the changes in the absorption coefficients at each node of our finite-element mesh. The reconstruction algorithm accounts for the path length between given source detector positions and reconstructs the predicted changes in the obsorption coefficient $\left(\Delta \mu_{a}\right)$ at each node. By solving Eqs $2 \mathrm{a}$ and $2 \mathrm{~b}$, the changes in oxyhemoglobin, $\Delta[H b O]$, and deoxyhemoglobin, $\Delta[H b]$, can be determined.

A trace of the measured output produced by the optical image system for the three Valsalva epochs is shown in Fig. 8a. Displayed are 15 traces of the normalized (to the rest period) measured-intensity profiles during three consecutive Valsalva maneuvers for source position \#3 at a wavelength of $760 \mathrm{~nm}$. It can be seen that the Valsalva maneuver protocol is very reproducible from epoch to epoch, and a strong signal drop in all detectors can be observed. At the peak of the Valsalva maneuver, the measurement intensities changed by up to $40 \%$ with respect to the rest period. To enhance the signal-to-noise ratio, a median filter of length 2 was used to smooth the intensity profile. For our 3D recon- 

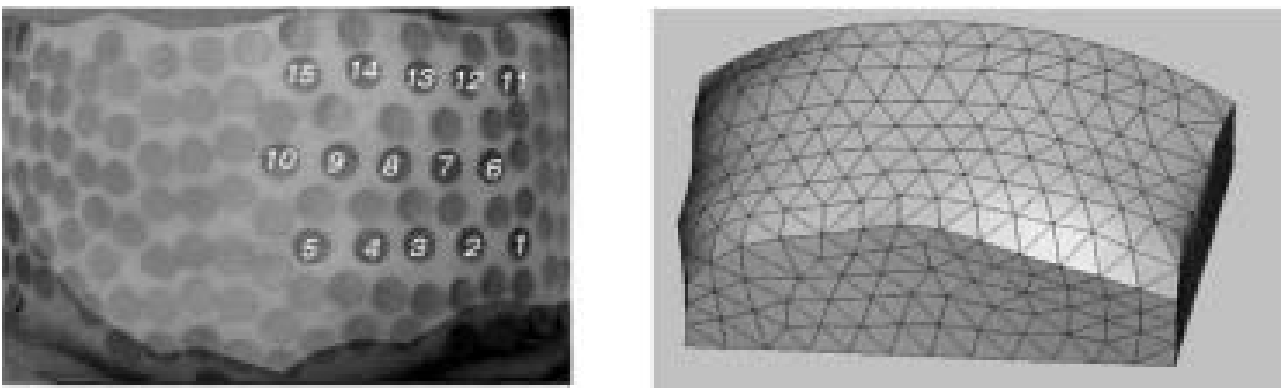

Fig. 7. (a-left): Placement of sources and detectors on forehead. The 15 source/detector positions are indicated by the encircled numbers. Other round dots indicate reference points used for the photogrammetric surface determination. The lighter shaded area depicts the outer surface of the finite-element mesh, which was used for the volumetric image reconstruction. (b-right): Mesh of forehead generated from photogrammetric data. Note that only a subset of surface notes is displayed. The actual calculations were performed on a finite-element mesh with 11,255 nodes, resulting in an average distance between nodes of $\sim 0.2 \mathrm{~cm}$.
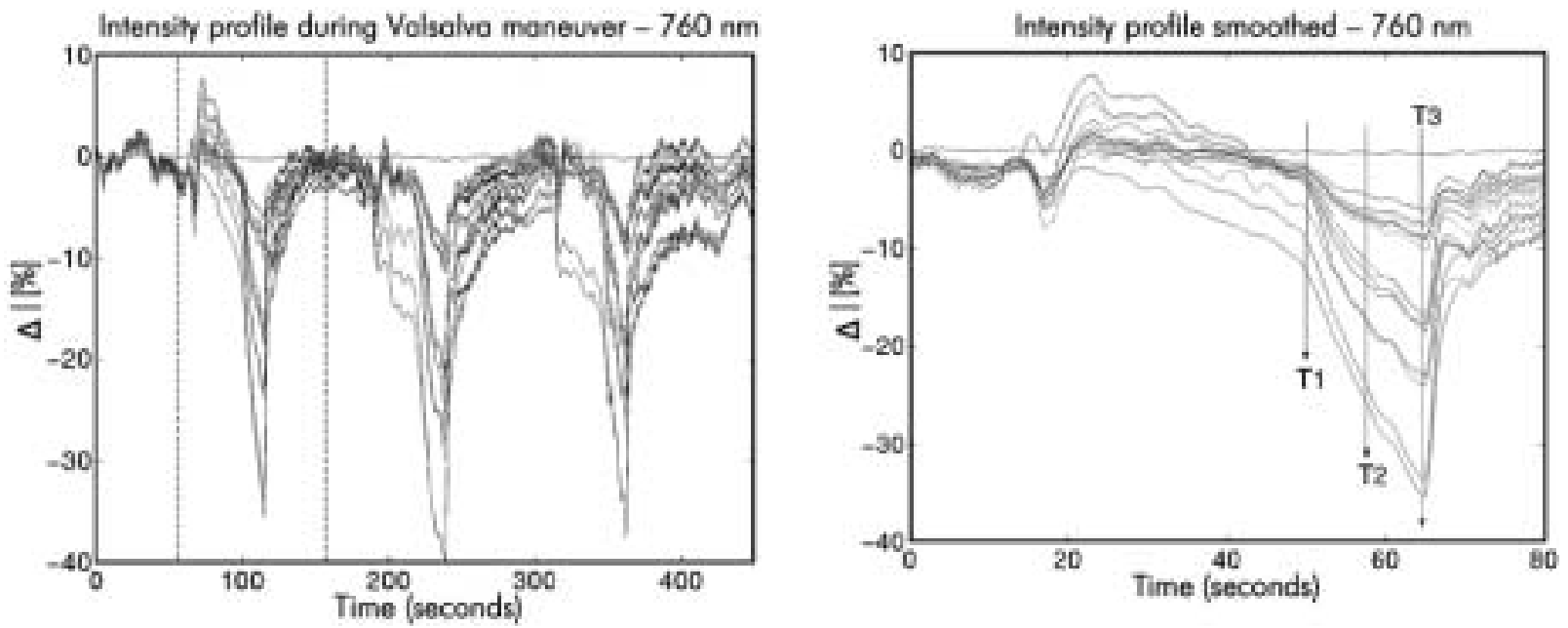

Fig. 8. (a-left): Time series of 15 detector readings during three successive Valsalva maneuvers. The source was located at position three (see Fig. 7). Each Valsalva maneuver was followed by a rest period. The measurements displayed were performed with near-infrared light at $\lambda=760 \mathrm{~nm}$. (b-right): Median-filtered time series for first Valsalva maneuver epoch seen in Fig. 8a.

structions, we focused on the data during the first of the three epochs, which are displayed in Fig. 8b. At $t=10$ seconds, the Valsalva maneuver began. Initially, an increase in signal was observed, which returned to baseline within 10-15 seconds. Then, the signal decreased steadily and approached a minimum at $t=65$ seconds, at which point the Valsalva maneuver was stopped. The signal recovers rapidly and eventually returns to baseline.

For the "difference" reconstruction, we used the ratio of the data at the time point T3 with respect to data at time point $t=0$ (Fig. 8b). The reconstruction was stopped after 25 iterations. At this point, no further changes in the reconstructed distributions were observed. The 25 iterations took approximately four hours on a Pentium III $550 \mathrm{Mhz}$ processor. Figure 9 shows the 3D distribution of the reconstructed values for changes in deoxyhemoglobin and oxyhemoglobin. Displayed are volumetric reconstructions for three different views: frontal (standing in front of body and looking toward the face), side (standing next to body and looking toward the right ear), and aerial (looking from the top of the head down toward the body). The different colors (shadings) represent isosurfaces of constant oxyhemoglobin and deoxyhemoglobin concentration changes relative to the reference point at $t=0$. One can see that two major regions of deoxygenation have evolved in which $\Delta H b$-values of up to $0.047 \mathrm{mM}$ can be found. Oxyhemoglobin values have changed by as much as $0.12 \mathrm{mM}$ in the center and upper right corner of the forehead. 

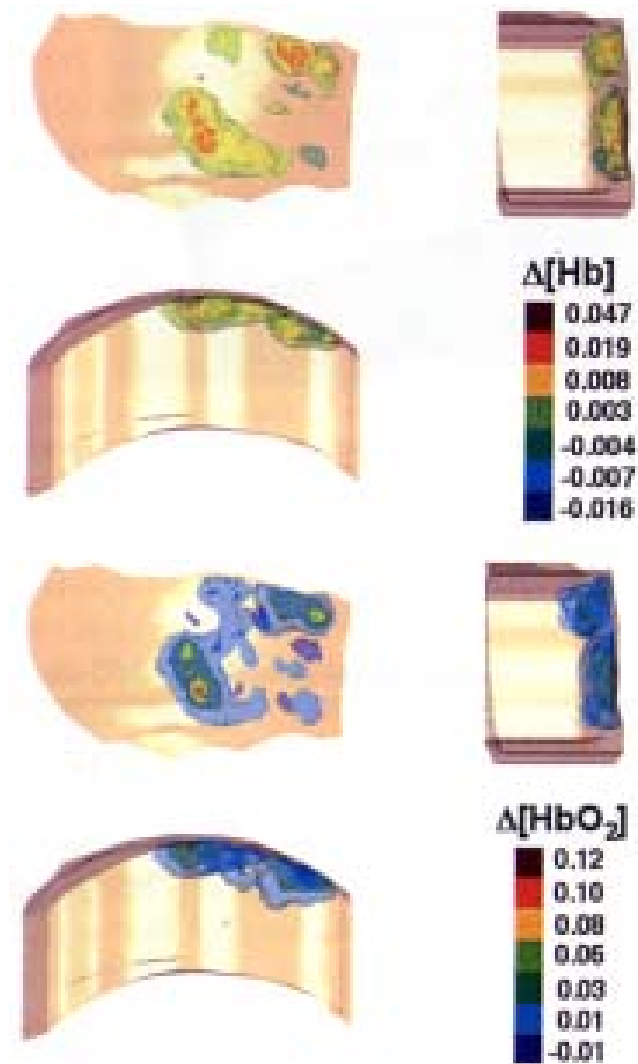

Fig. 9. Reconstructed changes in deoxyhemoglobin (top) and oxyhemoglobin (bottom) at time T3 (see Fig. 8b) during a Valsalva maneuver. The changes are given in units of $[\mathrm{mM}]$. Shown are a frontal view (upper left), a side view (upper right), and an aerial view (lower left) of the forehead.

\subsubsection{Diffusion vs. transport results}

The above-described results were obtained with a MOBIIR code that uses the diffusion equation as a forward model. As noted in Section 2.3, the diffusion model may not be a good model for light propagation in the brain, which contains low scattering regions that are filled with cerebrospinal fluid. To test the influence of the model, we used the same data and mesh to perform a reconstruction with a MOBIIR code that uses the equation of radiative transfer as a forward model [58]. The results are shown in Fig. 10. While one observes similarities in the overall spatial distribution, the values of the maximal changes differ substantially. The diffusion code finds maximal changes in deoxyhemoglobin of $0.047 \mathrm{mM}$, and the transport code finds $0.065 \mathrm{mM}$. Also, the center of this change is slightly shifted, which can be seen most clearly in the aerial view. The radiative-transfer code results located the maximal change approximately $0.5 \mathrm{~cm}$ deeper inside the head than did the diffusion analysis.
The time for a reconstruction of an oxy- or deoxyhemoglobin image using the diffusion code was approximately four hours on a Pentium III $550 \mathrm{Mhz}$ processor. This time includes the reconstruction of the absorption changes at two wavelengths for a mesh with 11,255 notes. The transport-theory-based code converged after 12 iterations, which took approximately 16 hours on the same workstation. The difference in the number of iterations required for convergence most likely resulted from the different optimization schemes that were used in the diffusion and transport algorithms. While the diffusion-based code employed a conjugate-gradient method that required complete line minimization, the radiative-transfer code used a Broyden-Fletcher-Goldfarb-Shanno (BFGS) minimization method [59] that did not require complete line searches.

There may be several reasons why the observed differences are not larger. First, it should be noted that only changes in optical and physiological properties were reconstructed. Pei et al. [118] recently showed that, in this case, the forward model does not play such an important role. Hillman et al. [119], however, reported in another study that, even for relative data, the accuracy of the forward model cannot be neglected. Another reason for the small difference could be the fact that the transport code was used with an isotropicscattering phase function instead of an anisotropicscattering phase function. Anisotropic-scattering phase functions require a fine angular discretization, which leads to increased memory requirements and computation times. Furthermore, the spatial discretization of $\sim 0.2 \mathrm{~cm}$ between adjacent notes may not be small enough to capture all of the effects. Finally, the expected effect of the cerebrospinal fluid layer may be smaller than expected. Additional studies are necessary to fully explore all of these aspects and to determine when the diffusion approximation suffices and when a transport-theory-based algorithm is required.

\subsection{Small-animal imaging}

Over the last 10 years, there has been an increasing interest in small-animal imaging systems. This interest is motivated by the progress in transgenic manipulation of small animals, which are prone to certain disease and pathological conditions. In studying these animals, it is possible to link specific genes to molecular, cellular, and organ functions during both good health and disease. Studies of the biochemistry and physiology of the brain, heart, musculosketetal, and metabolic systems 

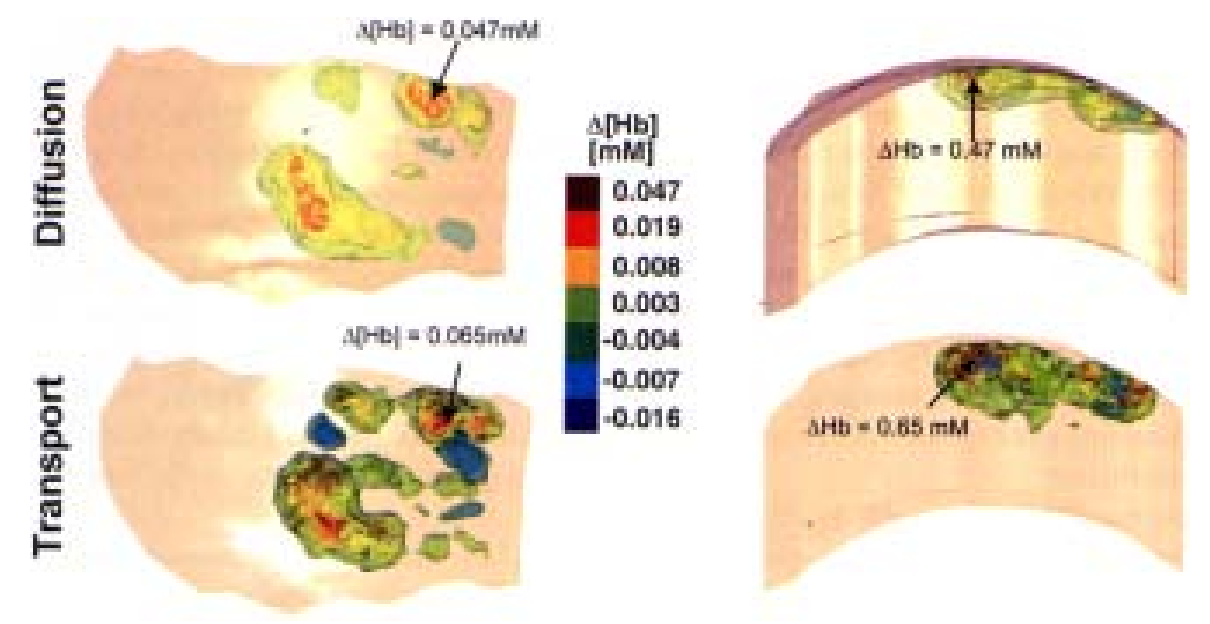

Fig. 10. Comparison of diffusion- and transport-based volumetric reconstruction results of dexoyhemoglobin distributions during a Valsalva maneuver. The top row shows diffusion results, with a head-on view on the left and an aerial view (from the top of the head) on the right. The bottom row shows the same views; however, an image-reconstruction code was used that is based on the equation of radiative transfer. While the overall patterns are similar, strong differences in the amplitude of the changes are visible. The maximum changes using the transport-based code are $0.65 \mathrm{mM}$, while the diffusion-based code finds maximal changes of only $0.47 \mathrm{mM}$.

historically has been through necropsy methods, which require the sacrificing of many animals. With the advent of new small-animal imaging systems, it has become possible to perform non-invasive assays to monitor the progression of diseases and biological processes in live small animals.

Commercial small-animal optical systems exist mainly for 2D surface imaging of fluorescent or bioluminescent probes [120-124]. Ntziachristos et al. [125] recently developed a first laboratory prototype for 3D fluorescence imaging. Although several commercial systems exist to monitor blood-oxygenation in humans, a dedicated optical system for tomographic 3D imaging of blood-related parameters and hemodynamics in small animals is not available yet. Several laboratory prototypes do exist, however. For example, Nemoto et al. conducted somatosensory stimulation studies in rats by stimulating the hind limb [126]. But instead of imaging through the skin and skull, the bone was made translucent using a carboxymethyl polymer, and only $2 \mathrm{D}$ tomographic maps were generated by taking images with a CCD camera. Siegel et al. [33], in a paper about a new SSD instrument, showed topographic maps of localized changes in cortical hemodynamics in response to somatosensory provocation in an anesthetized rat. The algorithm used was based on the diffusion theory for semi-infinite media. Cheung et al. [127] also generated topographical maps during hypercapnia experiments on rats. The maps were obtained with a frequency-domain code for semi-infinite media.
By combining the DYNOT imaging system described in Section 2.1.3 with our 3D MOBIIR scheme described in Sections 2.2.2. and 3.1.3, we have started to explore the possibilities of volumetric brain oximetry and hemodynamic monitoring in rats. Next, we present results concerning hypercapnia and paw-stimulation studies.

\subsubsection{Hypercapnia studies}

Adult male Sprague-Dawley or Wistar albino rats weighing approximately 300 gram were initially anesthetized with halothane (inhalation) and then injected with urethane $(1.5 \mathrm{~g} / \mathrm{kg})$ intraperionteally as a $20 \%$ solution in water (divided dose). Blood pressure was monitored via a polyethylene catheter inserted into one femoral artery (usually left). The intra-arterial tubing was connected to a blood-pressure transducer (CyQ or 1870-D43 Columbus Instruments, Columbus OH). A tracheotomy was performed through a ventral midline incision in the neck and a stainless steel t-tube was sutured in place. Animals typically were held ventral side up in a stereotaxic frame. The optical probe, consisting of four sources and 12 detectors (see Fig. 11a), was applied to the dorsal side of the head with a micromanipulator after shaving the skin. Animals were ventilated (2-5 cc stroke volume, 40 breaths per minute, resulting in $>95 \%$ oxygen saturation on room air as measured by pulse oximeter; Harvard Apparatus rodent respirator) throughout the experiment. After being connected to the ventilator, rats were paralyzed with decamethonium $(0.2 \mathrm{mg} / \mathrm{rat} \mathrm{ip})$. Specific mixtures of nitrogen, 
oxygen, and carbon dioxide were prepared using separate voltage-controlled mass-flow regulators (Dwyer GFC mass-flow controllers: 0-20 ml/min for $\mathrm{CO}_{2}, \mathrm{O}_{-}$ $200 \mathrm{ml} / \mathrm{min}$ for $\mathrm{O}_{2}$ and $0-1000 \mathrm{ml} / \mathrm{min}$ for $\mathrm{N}_{2}$ ). Gases were mixed in a $385-\mathrm{ml}$ chamber before being supplied to the ventilator (no rebreathing). Oxygen levels were fixed at $30 \%$, and changes in $\mathrm{CO}_{2}$ concentrations were compensated by changes in $\mathrm{N}_{2}$. The baseline gas mixture was $0.3 \% \mathrm{CO}_{2}, 30 \% \mathrm{O}_{2}$, and $69.7 \% \mathrm{~N}_{2}$. A perturbation lasted one to three minutes, with five to 30 minutes of baseline gas exposure occurring before a subsequent perturbation.

The raw experimental measurement data consisted of a time series for each source and each wavelength $(\lambda=$ $777 \mathrm{~nm}$ and $807 \mathrm{~nm}$ ). As an example, Fig. 11b displays 12 traces of detector readings during the protocol with a source at position number 2 (upper right in Fig. 11a). Each trace was normalized by calculating the mean of the measured intensity during the steady-state period and subsequently dividing all intensities in the trace by that mean. Furthermore, the original data was filtered, using a median filter, to enhance the signal-to-noise ratio. The black bar in the figure depicts the extent of the $\mathrm{CO}_{2}$ perturbation, and the arrows are the time points used for the reconstruction. At $808 \mathrm{~nm}$, an increase in intensity is observed during the first 10 seconds, followed by a drop in intensity. Similar curves were obtained at $777 \mathrm{~nm}$.

Using data from both wavelengths, it is possible to calculate oxy- and dexoyhemoglobin concentration changes as described in Section 3.1.1. For the volumetric difference reconstructions, we used the ratio of the measured intensity during various time points (black arrows labeled $\mathrm{T}_{1}-\mathrm{T}_{5}$ in Fig. 11b) with respect to the measured intensity during the rest-state, (red arrow in Fig. 11). These black arrows represent a snapshot of measured intensity for all source/detector combinations during consecutive instances of induced hypercapnia. For each snapshot, a volumetric reconstruction of $\Delta \mu_{a}$ and $\Delta \mu_{s}$ was performed, which resulted in three volumetric images of changes in oxyhemoglobin, deoxyhemoglobin, and total hemoglobin in the rat brain. To apply our MOBIIR scheme to the acquired data, a geometric model of the underlying tissue was generated. The imaging probe was modeled as a rectangular parallelepiped and converted into a 3D mesh that could be used within the context of our finite-element reconstruction algorithm. The resulting volume was $12 \times 7$ $\times 10 \mathrm{~mm}^{3}$ cube composed of 2830 nodes and 13,728 tetrahedral elements. Each reconstruction consisted of 25 iterations of a conjugate gradient scheme and took approximately one hour on a Pentium III $550 \mathrm{MHz}$ processor. A representative series of images of changes in oxyhemoglobin at times point $\mathrm{T} 1$ through $\mathrm{T} 5$ is displayed in Fig. 12. The shown coronal slices were cut midway through bregma and lambda (Fig. 11a). As one views the series, one can observe a steady increase in oxyhemoglobin, which is circumscribed and symmetrically located. It should be noted that, because SSD-type data was employed, some degree of cross talk between the $\mu_{a}$ and $\mu_{s}^{\prime}$ reconstructions could occur. Hence, some of the oxyhemoglobin and deoxyhemoglobin effects may be attributed to scatter changes.

\subsubsection{Functional stimulation studies}

For the functional stimulation studies, animals were prepared as described in Section 3.2.1. After paralysis, custom parallel bipolar stimulating electrodes $(0.1-\mathrm{mm}$ varnish-insulated stainless steel, acute conical tips, 0.5$\mathrm{mm}$ tip separation) were inserted into the foot pad. Isolated stimulus pulses $(0.5-\mathrm{ms}$ duration, $<0.5-\mathrm{mA}$ intensity, rates from 1 to $10 \mathrm{~Hz}$ ) were applied using a commercial stimulator (A\&M Systems 2100). Trains of stimuli lasted 10 to 30 seconds and were separated by equivalent times without stimulation.

Figure 13a shows the 12 detector readings (source \#2) at wavelengths $\lambda=760 \mathrm{~nm}$ during one episode of this stimulation. In this example, the stimulation started at $t=20$ seconds and lasted for 20 seconds. A moving average filter (width $=7$ data points) was applied to the data. We observed changes correlated to the stimulus in the detector readings of up to $1.5 \%$; at $832 \mathrm{~nm}$ (not shown), changes generally were smaller than $0.5 \%$ in the opposite direction.

The data from all source-detector pairs at $t=35$ seconds were used as input to our reconstruction code, which calculated the changes in oxy- and dexoyhemoglobin with respect to $t=0$ seconds. Figure $13 \mathrm{~b}$ shows the results of the deoxyhemoglobin calculations for three different slices through the brain. One can see clearly the lateralization of the effect as is expected from various functional MRI studies.

For this reconstruction, we used an anatomical prior for regularizing the reconstruction process. Using histological and gross anatomical specimens, we constructed a generalized model of the rat head (Fig. 14). We assigned different optical properties to different tissues of the head. During the reconstruction process, we updated the optical properties at all nodes inside the brain independently. On the other hand, skin, skull, and muscular tissue were treated as homogenous layers. The optical properties at notes within each layer were 

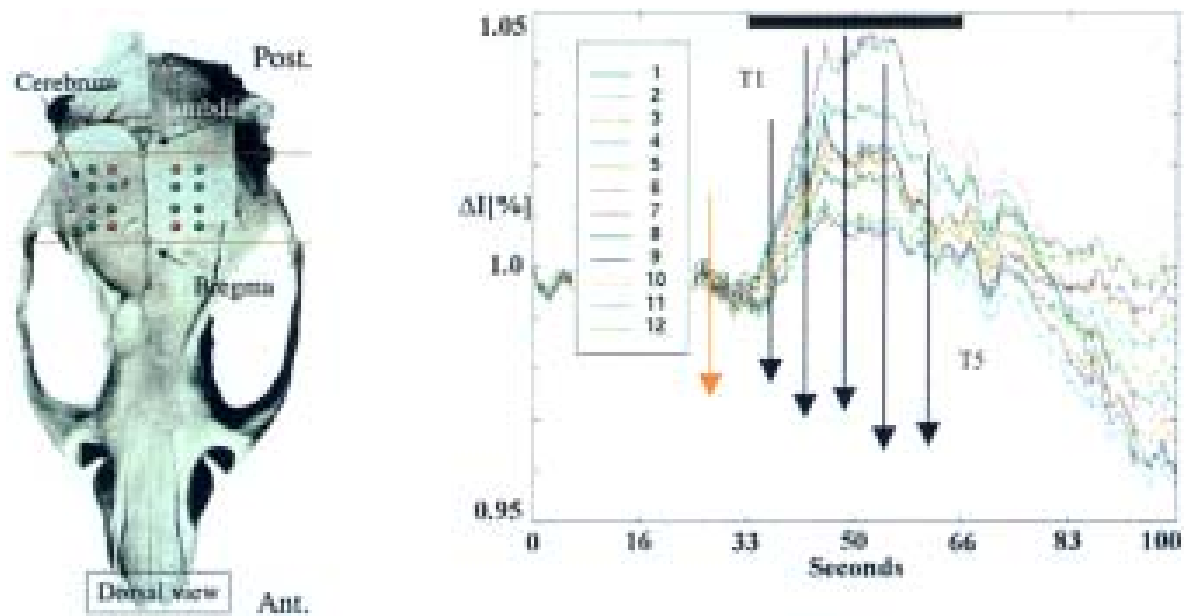

Fig. 11. (a-left): Positioning of sources (red circles) and detectors (blue circles) on the rat head relative to lambda and bregma. (b-right): Intensity change (normalized to rest at $t=0$ ) as a function of time for source 2 (upper right red circle in Fig. 11a) and all detectors at a wavelength of $\lambda=$ $808 \mathrm{~nm}$. Similar curves were obtained at $\lambda=777 \mathrm{~nm}$.
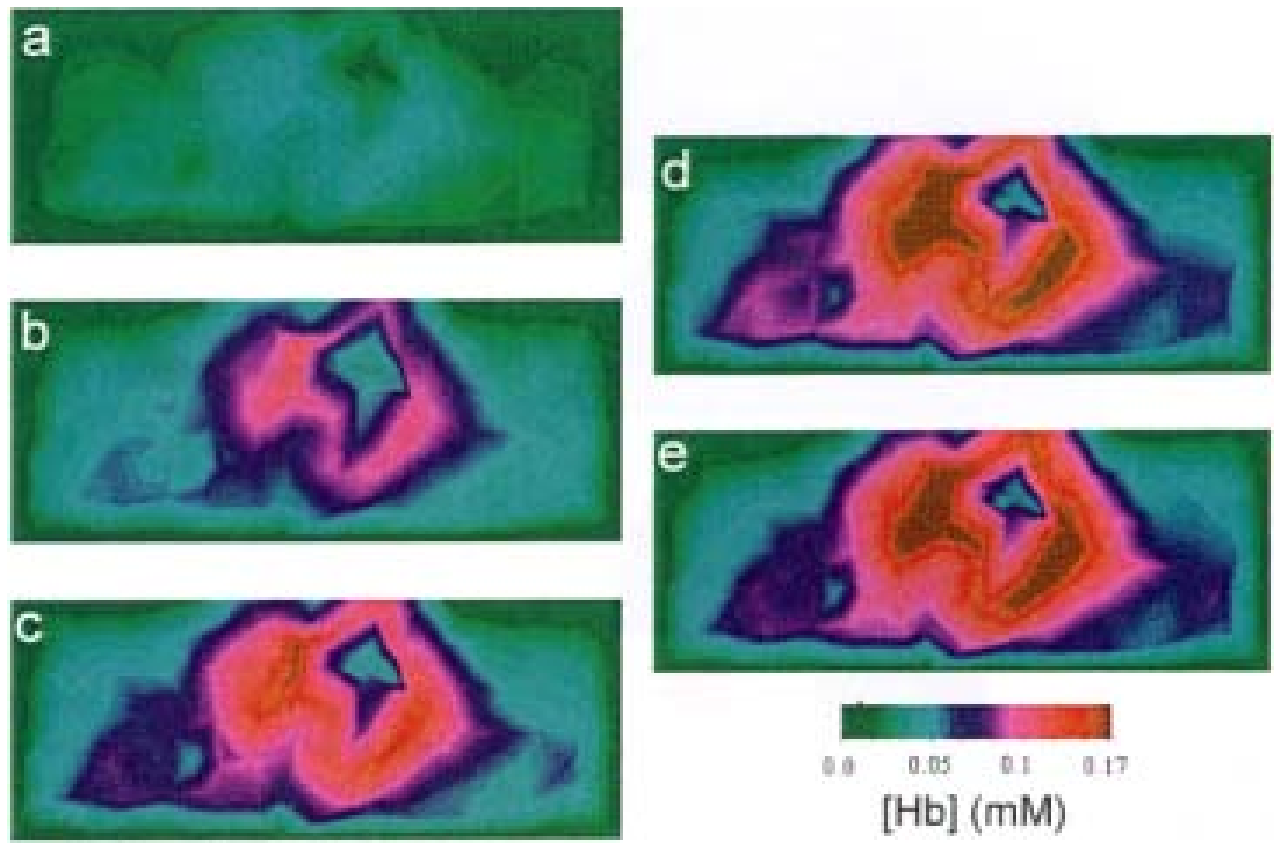

Fig. 12. Maps of deoxyhemoglobin changes for coronal sections through a rat brain. One can see an increase in deoxyhemoglobin as one progresses from T1 (a) through T5 (e).

not updated independently. Instead the optical properties at each note were changed by an average value for a given layer. We found that this considerably improved the image-reconstruction results and minimized the artifacts that often are encountered in the vicinity of the source and detectors. Previously, Pogue and Paulsen reached similar conclusions using a numerical model of the rat brain [128].

\subsection{Joint imaging}

Besides measuring hemodynamics and changes in various blood-related parameters, optical techniques also are quite sensitive to scattering changes in tissue. An example is the application of NIR diffuse-optical tomography to imaging and monitoring the progression of rheumatoid arthritis (RA). RA is a chronic, progressive, 

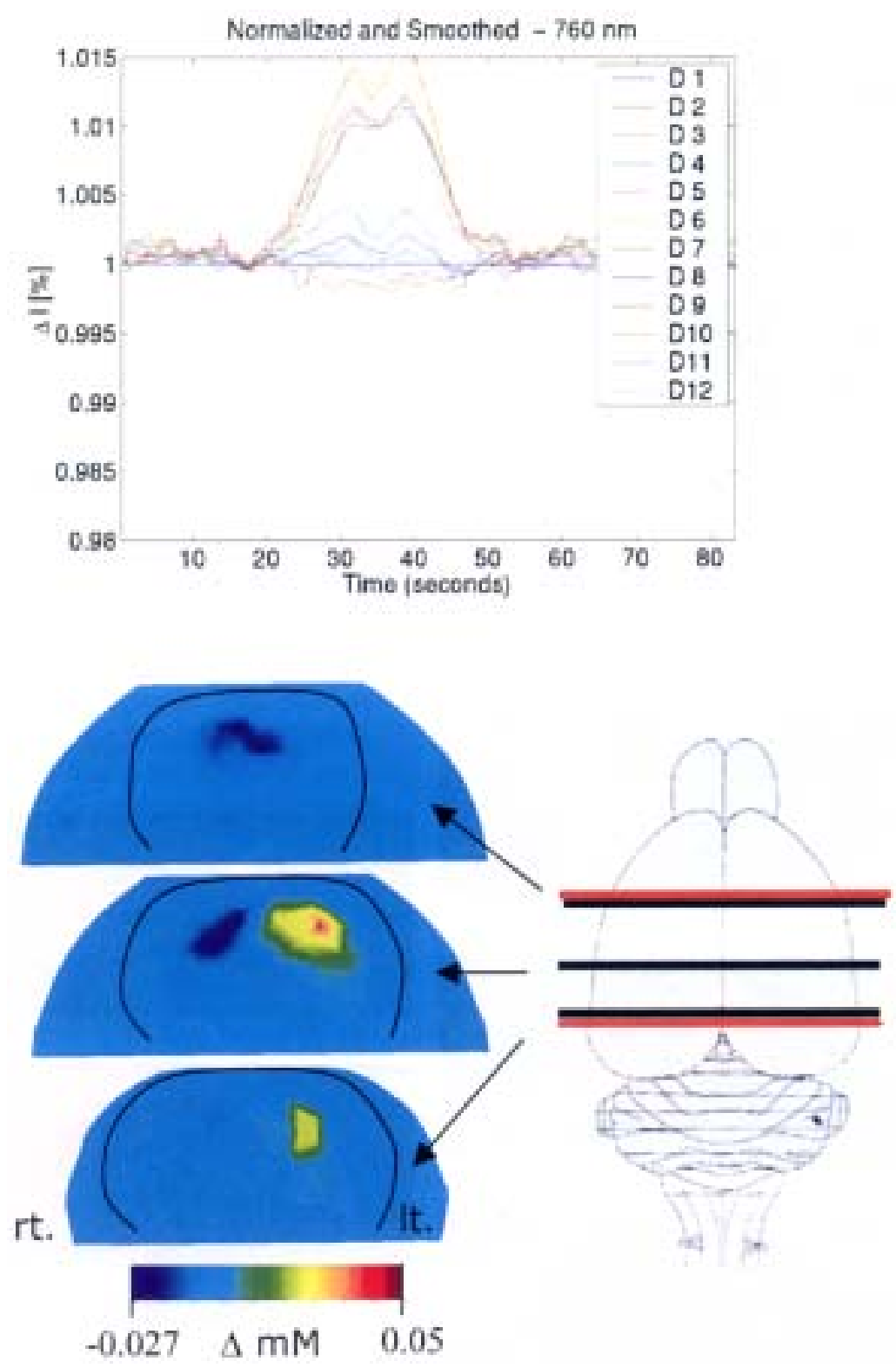

Fig. 13. (a-top): Time series for one source and all detectors at $\lambda=760 \mathrm{~nm}$. Depicted are the changes in normalized signal strength. Data from all sources and detectors at $t=35$ seconds were chosen for the volumetric reconstruction shown in Fig. 13b. (b-bottom): Reconstruction result for the hind-paw stimulation measurements, which shows the lateralized, hemispheric-specific response. Red lines indicate positions of lambda and bregma.

inflammatory disease that primarily attacks peripheral joints, surrounding tendons, and ligaments [129]. This disease, which often is associated with significant pain and disability, affects approximately 2.1 million people in the United States [130,131]. RA is characterized by an inflammatory synovitis that leads to cartilage and bone destruction and consequent loss of function. The synovial tissue becomes infiltrated with inflammatory cells and activated, invasive, fibroblast-like cells that may invade bone and cartilage. Four different disease stages, during which the tissue change is gradual, can be distinguished [132,133].

Imaging of the joint so far has played a role only in later stages of the disease. Radiography can document bone damage that results from RA and can visualize the narrowing of cartilage spaces. However, it has long 

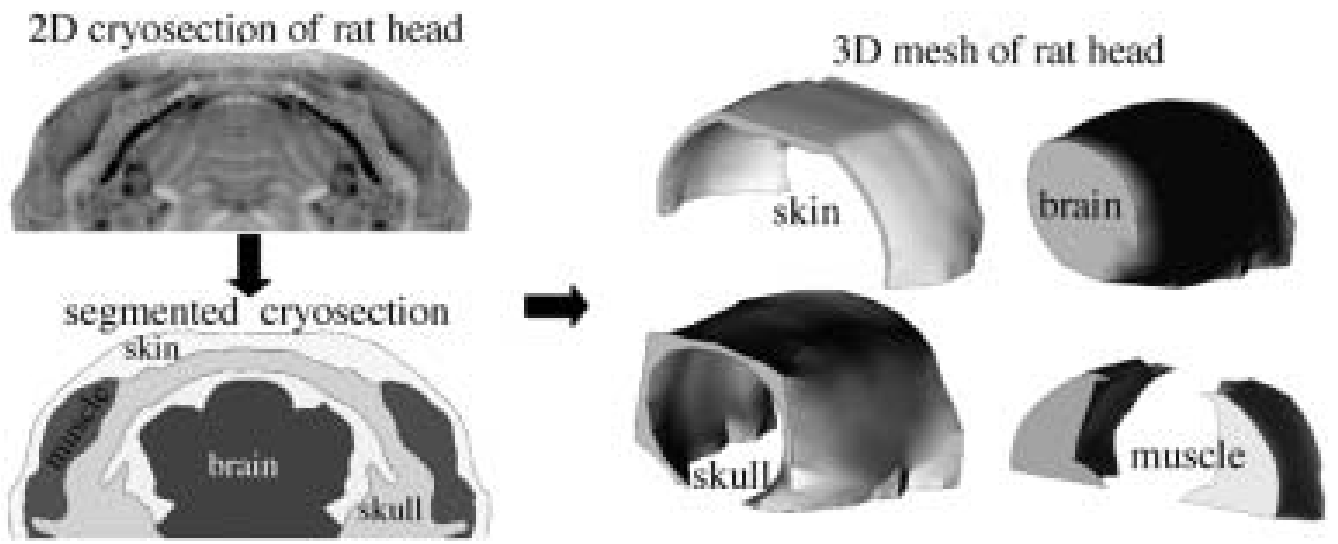

Fig. 14. Generation of an anatomically accurate finite-element mesh of a rat head.

been recognized that radiography is insensitive to the early manifestations of RA. Optical techniques promise to provide a new tool for the early detection of RA. Changes in the optical properties of the synovium and in the synovial fluid can be observed even in very early stages of the disease. For example, the normally clear, yellowish synovial fluid turns into a turbid, grayish substance [134].

After several numerical feasibility studies [135-137] and experiments involving tissue phantoms and healthy joints [138,139] showed that optical joint imaging is feasible, the goal of recent work has been to perform first clinical studies on human subjects with RA in the proximal-inter-phalangial (PIP) joint.

\subsubsection{Methods}

In diagnosing RA, it is particularly important to determine changes in the optical properties of the synovial fluid and the synovium. These changes are dominated by changes in the scattering coefficient. Obtaining these optical properties requires reconstructing absolute values rather than relying on dynamic changes. To this end, we developed a device that permits highaccuracy measurement of transmission profiles. A photograph and schematic of the device is shown in Fig. 15. A laser diode and a Si-photodiode are scanned independently over a distance of up to $3 \mathrm{~cm}$ along the saggital plane across the joint. The photodiode is in direct contact with the finger, while the beam of the laser diode is focused to a spot of approximately $0.3 \mathrm{~mm}$ in diameter on the back of the finger. During a measurement, the laser diode first is brought to the desired position on the back of the finger. The photodiode then is scanned over a distance of $3 \mathrm{~cm}$, and the transmitted intensities are detected. After the detector scan is finished, the laser diode is moved by a distance of $\Delta x$, the photodiode performs another scan, and so on. In this way, several transmission profiles are obtained. Because no optical fibers are used and the same source and detector are employed for each measurement, the recorded profiles can be compared directly to one another without additional calibrations for fiber-incoupling losses, different source strengths, or detector sensitivities. The measured data is then input to a MOBIIR scheme (see Section 2.2.2) that uses the equation of radiative transfer as a forward model.

\subsubsection{Results}

An example of a set of measurements is shown in Figs 16a and 16b. The subject was diagnosed with RA of the PIP joint of one hand, while the same joint in the contra-lateral hand was found to be unaffected. Optical trans-illumination measurements were performed on the same joint of both hands. In both figures, transillumination curves for five source positions, which are indicated by the arrows, are shown. Source position 2 is closest to the fingertip, and source position 18 is farthest from the fingertip. In Fig. 16a, which illustrates a measurement on a healthy joint, the trans-illumination data for source position 10 clearly shows the strongest transmission signal, with a maximal amplitude that is at least 1.5 times as strong as any other measurement at a different source position. This is expected because source position 10 is located directly on top of the joint, which is filled with relatively low-scattering and lowabsorbing synovial fluid. In Fig. 16b, which shows measurements on a rheumatoid joint, measurements at source position 10 show a weaker signal. Here, the strongest signal is measured at the source position that is closest to the fingertip, where the finger diameter is smallest. As the source is moved farther away from the fingertip, the signal becomes progressively smaller. 

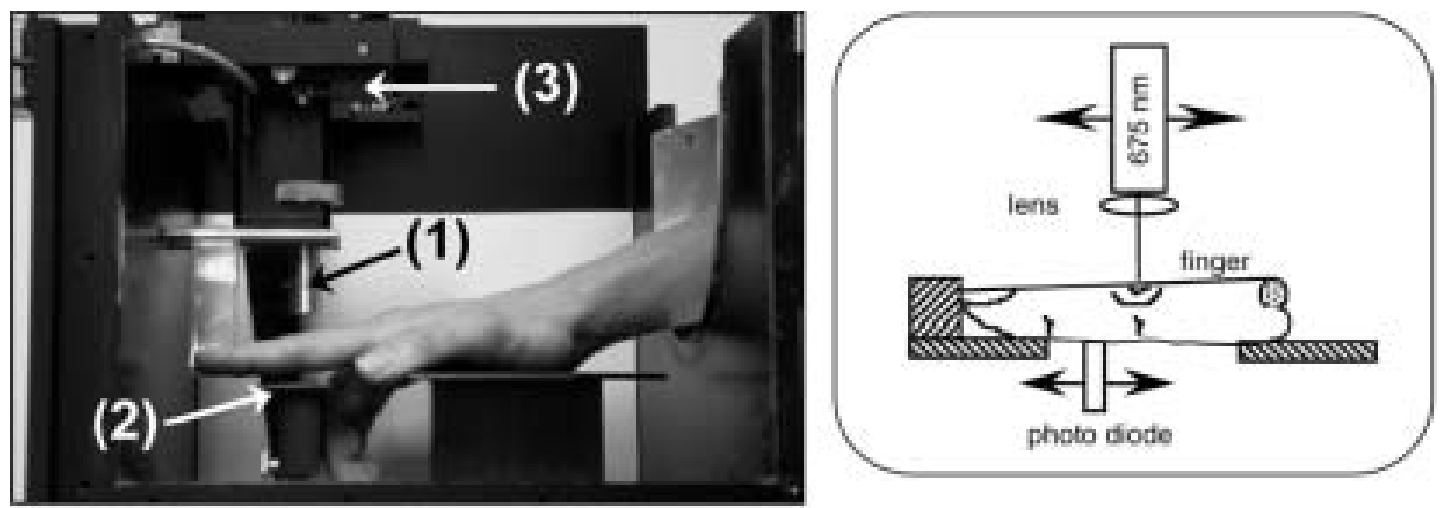

Fig. 15. Experimental set-up for sagittal joint imaging ((1) laser, (2) detector, (3) stepping motors)).
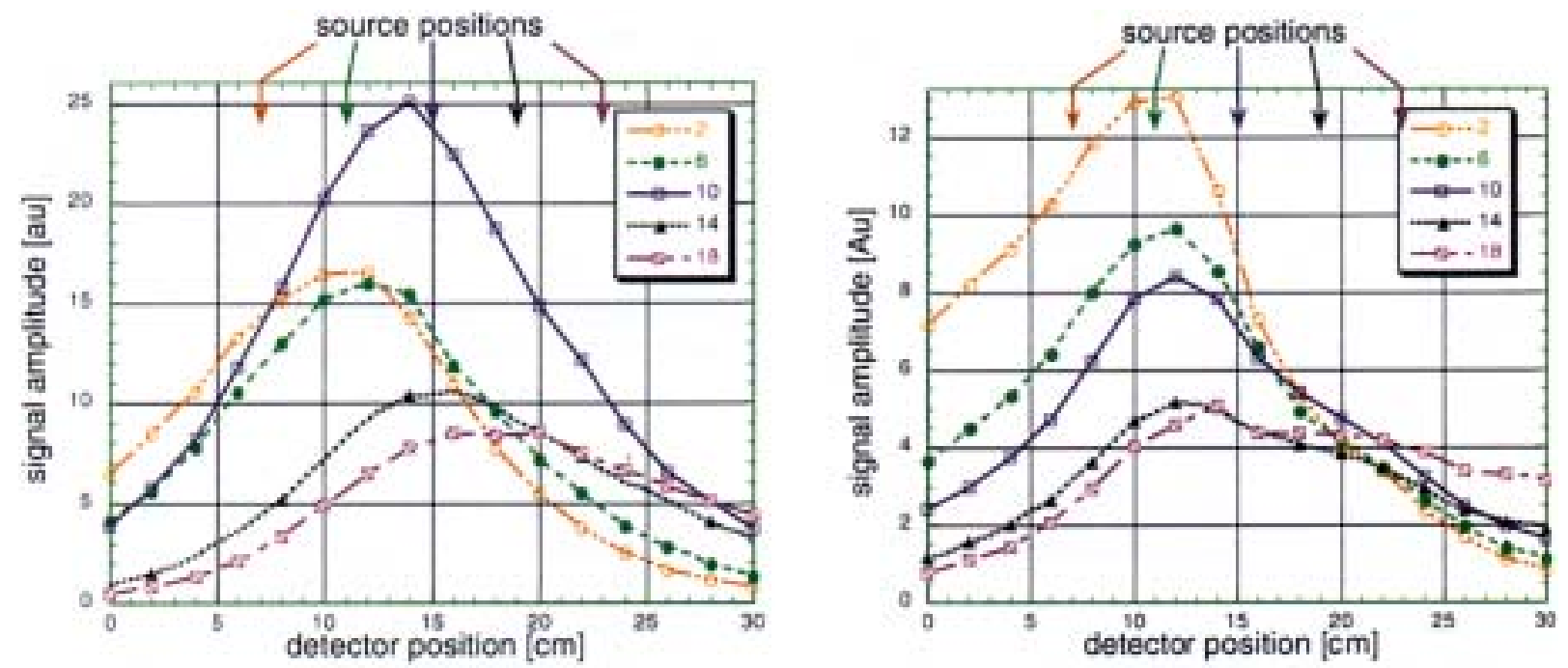

Fig. 16. Intensity transmission profiles for five different source positions for a healthy joint (left) and the same joint on the contra-lateral hand with rheumatoid arthritis (right). Source position 2 is closest to the fingertip, while source position 18 is farthest from the fingertip. Source position 10 is located directly above the PIP joint.

Figure 17 shows the reconstructed cross-section of the scattering coefficient in the saggital plane of the healthy joint (left) and the joint with RA (right). The fingertip is located to the right of the images, which show a 3-cm-wide sagittal section of the finger with the joint located in the center. The reconstruction was started with an initial guess of $\mu_{s}=100 \mathrm{~cm}^{-1}$ and $\mu_{a}=0.5 \mathrm{~cm}^{1}$. The anisotropy factor $\mathrm{g}$ was fixed at 0.9 . Although the spatial resolution is poor, the decrease in value of the optical properties around the location of the joint, which is filled with the low-scattering and low-absorbing synovial fluid, is clearly visible. In the reconstructions of the joint affected with RA, this decrease is much less pronounced. These results illustrate the potential of optical techniques to diagnose RA in its early stages, as other imaging modalities are not capable of measuring scattering changes in the synovial fluid. Clinical studies involving approximately 50 volunteers currently are underway in our laboratories to fully explore this potential. In addition to imaging changes in the scattering coefficient, tomographic imaging of hemodynamic processes in the vasculature of the joint also may be of diagnostic value, as recently was reported by Lasker et al. [140].

\subsection{Other applications}

There are several other promising applications of optical tomographic imaging. For example, Watanabe et al. [44] recently developed an optical system for pin- 

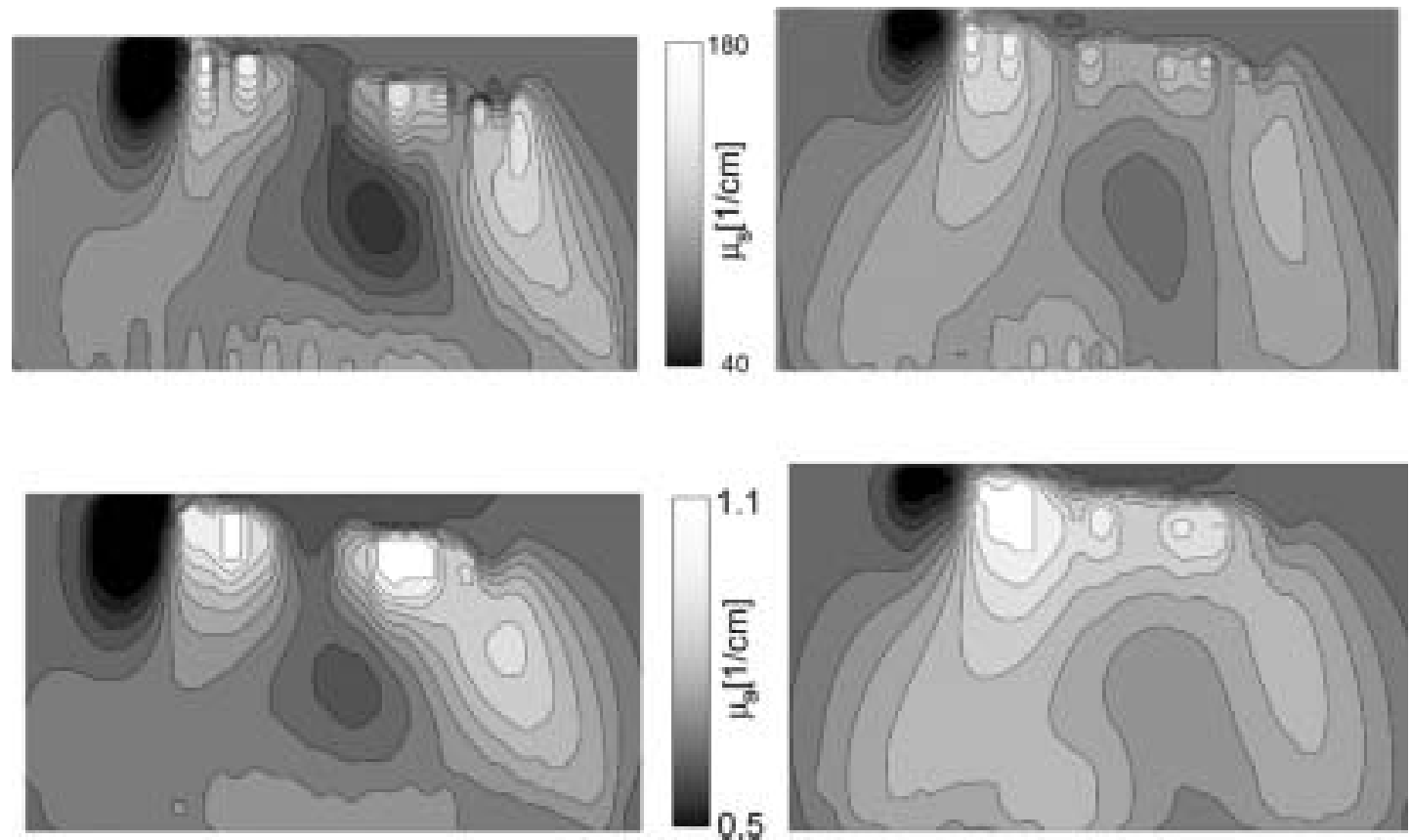

Fig. 17. Reconstructed cross-section of the scattering coefficient (top) and absorption coefficient (bottom) in the sagittal plane through the healthy joint (left) and the RA joint (right). The fingertip is located to the right of the images that show a 3-cm-wide section of the finger with the joint located in the center.

pointing the location of epileptic foci. Considerable effort has also been directed toward developing an optical imaging method for the early detection of breast cancer [25-27,141-146]. Although major advances have been made, initial clinical trials still are inconclusive and more work must be done to make optical breast imaging a standard clinical tool. Also of special interest here is the combination of MRI and optical imaging methods [81,147].

Another area of strong interest is the development of optical molecular-imaging methods (see e.g. [120125,148-151]). The field of medical imaging currently is undergoing a revolution. The advent of novel biochemical markers that can detect faulty genes and other molecular processes that precede the development of disease makes it possible for the first time to detect disease long before actual phenotypical symptoms appear. Up to now, major progress has been achieved in small-animal imaging. Since the early 1990s, research in this direction has been motivated by the substantial resources provided by the National Institutes of Health $(\mathrm{NIH})$ for the development of small-animal models for human diseases such as cancer, diabetes, heart disease, and arthritis. These animal models provide a medium for studies that concern disease development, progression, and treatment. Molecular mark- ers have become increasingly important in these studies because, together with various imaging modalities, these markers allow the study of disease-related processes without sacrificing the animal. A good overview of this field recently was presented by Weissleder et al. [138].

\section{Summary}

DOT currently is developing into a viable addition to existing biomedical-imaging modalities, such as ultrasound, CT, MRI, PET, and SPECT. This novel imaging technique uses NIR light (approximately $700 \mathrm{~nm}$ $<\lambda<900 \mathrm{~nm}$ ) to probe the absorption and scattering properties of tissue. Recent technological and computational advances have led to first clinical trials involving brain, breast, and joint imaging.

The instrumentation used for DOT can be divided into three categories: SSD systems, FD devices, and time-domain TD instrumentation. Although TD measurements and analysis schemes theoretically provide the best differentiation between scattering and absorption coefficients, most commercially available systems are of the SSD or FD type. Such systems are comparatively easy to assemble, inexpensive, and allow 
for faster data-acquisition rates. SSD systems are particularly well suited for studying fast physiological changes, such as hemodynamic effects in functional brain imaging.

The image-reconstruction problem in DOT suffers from the strong scattering of near-infrared photons in biological tissue. Unlike in X-ray-based imaging, the probing light does not propagate on a straight line from the source to the detector. Therefore, traditional CT backprojection algorithms have limited applicability. To obtain more accurate images, so-called MOBIIR codes now are widely applied. These schemes computationally are much more demanding than are backprojection methods, but they account more rigorously for the underlying physics of light propagation in tissue.

A major application of DOT is brain imaging. By determining the spatially resolved distribution of bloodderived parameters such as oxy- and deoxyhemoglobin, researchers have started to explore the possibility of optical-functional imaging as well as stroke imaging and monitoring. To illustrate how optical data can be used to obtain 3D information about hemodynamic processes in the brain, we presented reconstructions of various blood parameters during a Valsalva maneuver. To validate the results obtainable with this new technology, we currently are pursuing studies in small animals. This allows for tighter control of various experimental parameters. Small-animal imaging studies draw further attention because of recent advances in molecular imaging. Molecular markers are under development that promise the detection of diseases on a biomolecular level long before phenotypic symptoms appear. Most of these markers currently are being tested in smallanimal studies, and optical techniques promise to play an important role in this field.

Beyond absorption changes due to variations in hemoglobin concentrations and other chromophores, optical methods also are sensitive to changes in the scattering properties of tissues. While the detectability of scattering changes in activated neurons through the skull by optical means still is being debated, it has been shown that scattering changes in the synovial fluid of patients with RA can be detected optically. The ultimate usefulness of near-infrared diffuse optical tomography for the diagnosis of arthritis as well as many other diseases still must be evaluated in larger clinical trials, which currently are ongoing.

\section{Acknowledgments}

We would like to acknowledge the advice and support concerning the DYNOT imaging systems from
Professor R. Barbour, SUNY Downstate Medical Center, Brooklyn, NY; and Dr. C. Schmitz, NIRx, LLC, Glen Head, NY. This work was supported in part by National Institute of Arthritis and Musculoskeletal and Skin Diseases (NIAMS) Grant Number R01 AR4625502: a Small Business Innovation Research (SBIR) grant (2R44-HL-61057-02) from the National Heart, Lung, and Blood Institute (NHLBI); the Whitaker Foundation for Biomedical Engineering; and the New York City Council Speaker's Fund for Biomedical Research: Toward the Science of Patient Care.

\section{References}

[1] B. Chance, R.R. Alfano, B.J. Tromberg, M. Tamura and E.M. Sevick-Muraca, eds, Optical tomography and spectroscopy of tissue IV, Proc. 4250 SPIE-The International Society for Optical Engineering, Bellingham, WA, (2001).

[2] B. Chance, R.R. Alfano and B.J. Tromberg, eds, Optical tomography and spectroscopy of tissue III, Proc. 3597 SPIEThe International Society for Optical Engineering, Bellingham, WA, (1999)

[3] B. Chance and R.R. Alfano, eds, Optical tomography and spectroscopy of tissue: theory, instrumentation, model, and human studies, Proc. 2979 SPIE-The International Society for Optical Engineering, Bellingham, WA, (1997).

[4] B. Chance and R.R. Alfano, eds, Optical tomography, photon migration and spectroscopy of tissue and model media: theory, human studies, and instrumentation, Proc. 2389, Part 1 and 2 SPIE-The International Society for Optical Engineering, Bellingham, WA, (1995).

[5] G. Müller, B. Chance, R.R. Alfano, S. Arridge, J. Beuthan, E. Gratton, M. Kaschke, B. Masters, S. Svanberg and P. van der Zee, Medical optical tomography: functional imaging and monitoring, Vol. IS11, SPIE Institutes for Advanced Optical Technologies Series, SPIE-The International Society for Optical Engineering, Bellingham, WA, (1993).

[6] A.J. Welch and M.J.C. van Gemert, Optical-thermal response of laser-irradiated tissue, New York: Plenum, 1995.

[7] J.R. Mourant, M. Canpolat, C. Brocker, O. Esponda-Ramos, T.M. Johnson, A. Matanock, K. Stetter and J.P. Freyer, Light scattering from cells: the contribution of the nucleus and the effects of proliferative status, J Biomed Opt 5(2) (2000), 131-137.

[8] J.R. Mourant, A.H. Hielscher, A.A. Eick, T.M. Johnson and J.P. Freyer, Evidence of intrinsic differences in the light scattering properties of tumorigenic and nontumorigenic cells, Cancer Cytol 84(6) (1998), 366-374.

[9] J.R. Mourant, J.P. Freyer, A.H. Hielscher, A.A. Eick, D. Shen and T.M. Johnson, Mechanisms of light scattering from biological cells relevant to noninvasive optical-tissue diagnostics, Appl Opt 37(16) (1998), 3586-3593.

[10] J.C. Hebden, S.R. Arridge and D.T. Delpy, Optical imaging in medicine.1. Experimental techniques, Phys Med Biol 42(5) (1997), 825-840.

[11] M.S. Patterson, B. Chance and B.C. Wilson, Time resolved reflectance and transmittance for the noninvasive measurement of tissue optical properties, Appl Opt 28 (1989), 22312336 . 
[12] D.V. O'Connor and D. Phillips, Time-correlated single photon counting, Academic Press, Orlando, FL, 1984.

[13] H. Liu, M. Miwa, B. Beauvoit, N.G. Wang and B. Chance, Characterization of absorption and scattering properties of small volume biological samples using time-resolved spectroscopy, Anal Biochem 213 (1993), 378-385.

[14] V. Ntziachristos, X.H. Ma, A.G. Yodh and B. Chance, Multichannel photon counting instrument for spatially resolved near infrared spectroscopy, Rev Sci Instrum 70(1) (1999), 193-201, Part 1.

[15] F.E.W. Schmidt, M.E. Fry, E.M.C. Hillman, J.C. Hebden and D.T. Delpy, A 32-channel time-resolved instrument for medical optical tomography, Rev Sci Instrum 71(1) (2000), 256-265.

[16] M.S. Patterson, J.D. Moulton, B.C. Wilson, K.W. Berndt and J.R. Lakowicz, Frequency-domain reflectance for the determination of scattering and absorption properties of tissue, Appl Opt 30 (1991), 4474-4476.

[17] S.J. Madsen, E.R. Anderson, R.C. Haskell and B.J Tromberg, Portable high-bandwidth, frequency-domain photon migration instrument for tissue spectroscopy, Opt Lett 19(23) (1994), 1934-1936.

[18] T.H. Pham, O. Coquoz, J.B. Fishkin, E. Anderson and B.J. Tromberg, Broad bandwidth frequency domain instrument for quantitative tissue optical spectroscopy, Rev Sci Instrum 71(6) (2000), 2500-2513.

[19] S. Fantini, M.A. Franceschini, J.S. Maier, S.A. Walker, B. Barbieri and E. Gratton, Frequency-domain multichannel optical detector for non-invasive tissue spectyroscopy and oximetry, Opt Eng 34(1) (1995), 32-42.

[20] R.D. Spencer and G. Weber, Measurement of subnanosecond fluorescence lifetimes with a cross-correlation phase fluorometer, Ann NY Acad Sci 158 (1969), 361.

[21] E. Gratton and M. Limkeman, A continuously variable frequency cross-correlation phase fluorometer with picosecond resolution, Biophys J 44 (1983), 315-324.

[22] J.R. Lakowicz and B.P. Maliwal, Construction and performance of a variable-frequency phase-modulation fluorometer, Biophys Chem 21 (1985), 61-78.

[23] B.A. Feddersen, D.W. Piston and E. Gratton, Digital parallel acquisition in frequency domain fluorimetry, Rev Sci Instrum 60 (1989), 2929-2936.

[24] M.A. Franceschini, K.T. Moesta, S. Fantini, G. Gaida, E. Gratton, H. Jess, W. Mantulin, M. Seeber, P.M. Schlag and M. Kaschke, Frequency-domain techniques enhance optical mammography: initial clinical results, Proc Natl Acad Sci USA 94(12) (1997), 6468-6473.

[25] B.W. Pogue, S.P. Poplack, T.O. McBride, W.A. Wells, K.S. Osterman, U.L. Österberg and K.D. Paulsen, Quantative Hemoglobin Tomography with Diffuse Near-Infrared Spectroscopy: Pilot Results in the Breast, Radiology 218(1) (2001), 261-266.

[26] T.O. McBride, B.W. Pogue, S. Jiang, U.L. Osterberg and K.D. Paulsen, A parallel-detection frequency-domain nearinfrared tomography system for hemoglobin imaging of the breast in vivo, Rev Sci Instrum 72(3) (2001), 1817-1824.

[27] A.E. Cerussi, A.J. Berger, F. Bevilacqua, N. Shah, D. Jakubowski, J. Butler, R.F. Holcombe and B.J. Tromberg, Sources of absorption and scattering contrast for nearinfrared optical mammography, Acad Radiol 8(3) (2001), 211-218

[28] D.M. Hueber, M.A. Franceschini, H.Y. Ma, Q. Zhang, J.R. Ballesteros, S. Fantini, D. Wallace, V. Ntziachristos and B. Chance, Non-invasive and quantitative near-infrared haemoglobin spectrometry in the piglet brain during hypoxic stress, using a frequency-domain multidistance instrument, Phys Med Biol 46(1) (2001), 41-62.

[29] M. Franceschini, V. Toronov, M.E. Filiaci, E. Gratton and S. Fantini, On-line optical imaging of the human brain with 160-ms temporal resolution, Opt Express 6(3) (2000), 49-57.

[30] C.H. Schmitz, M. Löcker, J.M. Lasker, A.H. Hielscher and R.L. Barbour, Instrumentation for fast functional optical tomography, Rev Sci Instrum 73(2) (2002), 429-439.

[31] C.H. Schmitz, M. Löcker, J.M. Lasker, A.H. Hielscher and R.L. Barbour, Performance characteristics of a siliconphotodiode-based instrument for fast functional optical tomography, in: Optical Tomography and Spectroscopy of Tissue IV, B. Chance, R.R. Alfano, B. Tromberg, M. Tamura, E.M. Sevick-Muraca, eds, SPIE-Proceedings 4250, Bellingham, WA, 2001, pp. 171-179.

[32] C.H. Schmitz, H.L. Graber, H.B. Luo, I. Arif, J. Hira, Y.L. Pei, A. Bluestone, S. Zhong, R. Andronica, I. Soller, N. Ramirez, S.L.S. Barbour and R.L. Barbour, Instrumentation and calibration protocol for imaging dynamic features in dense-scattering media by optical tomography, Appl Opt 39(34) (2000), 6466-6486.

[33] A.M. Siegel, J.J.A. Marota and D.A. Boas, Design and evaluation of a continuous-wave diffuse optical tomography system, Opt Express 4(8) (1999), 287-298.

[34] S.B. Colak, M.B. van der Mark, G.W. Hooft, J.H. Hoogenraad, E.S. van der Linden and F.A. Kuijpers, Clinical optical tomography and NIR spectroscopy for breast cancer detection, IEEE J Sel Top Quantum Electron 5(4) (1999), 11431158.

[35] Y. Yamashita, A. Maki and H. Koizumi, Measurement system for noninvasive dynamic optical topography, J Biomed Opt 4(4) (1999), 414-417.

[36] D.A. Benaron, D.C. Ho, S. Spilman, J.P. van Houten and D.K. Stevenson, Non-recursive linear algorithms for optical imaging in diffusive media, Adv Exp Med Biol 361 (1994), 215-222.

[37] S.A. Walker, S. Fantini and E. Gratton, Image reconstruction by backprojection from frequency domain optical measurements in highly scattering media, Appl Opt 36(1) (1997), 170-179.

[38] S.B. Colak, D.G. Papaioannou, G.W. Hooft, M.B. van der Mark, H. Schomberg, J.C.J. Paasschens, J.B.M. Melissen and N.A.A.J. van Asten, Tomographic image reconstruction from optical projections in light-diffusing media, Appl Opt 36(1) (1997), 180-213.

[39] M.S. Patterson, B. Chance and B.C. Wilson, Time resolved reflectance and transmittance for the noninvasive measurement of tissue optical properties, Appl Opt 28 (1989), 22312336

[40] J.A. Parker, Image Reconstruction in Radiology, CRC Press, Boca Raton, FL, 1990.

[41] R.L. Barbour, H.L. Graber, J.W. Chang, S.L.S. Barbour, P.C. Koo and R. Aronson, MRI-guided optical tomography: prospects and computation for a new imaging method, IEEE Comput Sci Eng 2(4) (1995), 63-77.

[42] M.A. Oleary, D.A. Boas and A.G. Yodh, Experimental images of heterogeneous turbid media by frequency-domain diffusing photon tomography, Opt Lett 20(5) (1995), 426428.

[43] Y. Hoshi, I. Oda, Y. Wada, Y. Ito, Y. Yamashita, M. Oda, K. Ohta, Y. Yamada and M. Tamura, Visuospatial imagery is a fruitful strategy for the digit span backward task: a study with 
near-infrared optical tomography, Brain Res Cogn Brain Res 9 (2000), 339-342.

[44] E. Watanabe, A. Maki, F. Kawaguchi, Y. Yamashita, H. Koizumi and Y. Mayanagi, Noninvasive cerebral blood volume measurement during seizures using multichannel near infrared spectroscopic topography, JBiomed Opt 5(3) (2000), 287-290.

[45] S.R. Arridge, Optical tomography in medical imaging, Inverse Probl 15 (1999), R41-R93.

[46] M. Schweiger and S.R. Arridge, A system for solving the forward and inverse problems in optical spectroscopy and imaging, in: Advances in Optical Imaging and Photon Migrations, OSA Trends in Optics and Photonics Series, vol. 2, R.R. Alfano and J.G. Fujimoto, eds, Optical Society of America, Washington, DC, 1996, pp. 263-268.

[47] K.D. Paulsen and H. Jiang, Spatially varying optical property reconstruction using a finite element diffusion equation approximation, Med Phys 22 (1995), 691-701.

[48] K.D. Paulsen and H. Jiang, Enhanced frequency domain optical image reconstruction in tissues through total variation minimization, Appl Opt 35 (1996), 3447-3458.

[49] H.B. Jiang, K.D. Paulsen, U.L. Osterberg, B.W. Pogue and M.S. Patterson, Optical image reconstruction using frequency domain data: simulations and experiments, J Opt Soc Am A 13(2) (1996), 253-266.

[50] R. Roy and E.M. Sevick-Muraca, Active constrained truncated Newton method for simple-bound optical tomography, J Opt Soc Am A 17(9) (2000), 1627-1641.

[51] S.S. Saquib, K.M. Hanson and G.S. Cunningham, Modelbased image reconstruction from time-resolved diffusion data, Proc. 3034, SPIE-The International Society for Optical Engineering, 1997, pp. 369-380.

[52] R.L. Barbour, H.L. Graber, J.W. Chang, S.J.S. Barbour, P.C. Koo and R. Aronson, MRI-guided optical tomography: prospects and computation for a new imaging method, IEEE Comput Sci Eng 2(4) (1995), 63-77.

[53] Y.Q. Yao, Y. Wang, Y.L. Pei, W.W. Zhu and R.L. Barbour, Frequency-domain optical imaging of absorption and scattering distributions by born iterative method, J Opt Soc Am A 14(1) (1997), 325-342.

[54] A.H. Hielscher, A.D. Klose and K.M. Hanson, Gradientbased iterative image reconstruction scheme for timeresolved optical tomography, IEEE T Med Imaging 18 (1999), 262-271.

[55] A.D. Klose and A.H. Hielscher, Iterative reconstruction scheme for optical tomography based on the equation of radiative transfer, Med Phys 26(8) (1999), 1698-1707.

[56] A.D. Klose, U. Netz, J. Beuthan and A.H. Hielscher, Optical tomography using the time-independent equation of radiative transfer, Part 1: Forward model, J Quant Spectrosc Radiat Transf 72(5) (2002), 691-713.

[57] A.D. Klose and A.H. Hielscher, Optical tomography using the time-independent equation of radiative transfer. Part 2: Inverse model, J Quant Spectrosc Radiat Transf 72(5) (2002), 715-732.

[58] G. Abdoulaev and A.H. Hielscher, Three-dimensional optical tomography with the equation of radiative transfer, J Electron Imaging (in press).

[59] A.K. Klose and A.H. Hielscher, Quasi-Newton methods in optical tomographic imaging, Inverse Probl 19 (2003), 387409.

[60] S.R. Arridge and W.R.B. Lionheart, Nonuniqueness in diffusion-based optical tomography, Opt Lett 23(11) (1998), 882-884.
[61] Y.L. Pei, H.L. Graber and R.L. Barbour, Normalizedconstraint algorithm for minimizing inter-parameter crosstalk in DC optical tomography, Opt Express 9(2) (2001), 97-109.

[62] N. Iftimia and H.B. Jiang, Quantitative optical image reconstruction of turbid media by use of direct-current measurements, Appl Opt 39(28) (2000), 5256-5261.

[63] Y. Xu, X.J. Gu, T. Khan and H.B. Jiang, Absorption and scattering images of heterogeneous scattering media can be simultaneously reconstructed by use of dc data, Appl Opt 41(25) (2002), 5427-5437.

[64] H.B. Jiang, N.V. Iftimia, Y. Xu, J.A. Eggert, L.L. Fajardo and K.L. Klove, Near-infared optical imaging of the breast with model-based reconstruction, Acad Radiol 9(2) (2002), 186-194.

[65] B.W. Pogue, S.P. Poplack, T.O. McBride, W.A. Wells, W.A. Osterman, U.L. Osterberg and K.D. Paulsen, Quantitative hemoglobin tomography with diffuse near-infared spectroscopy: pilot results in the breast, Radiology 218 (2001), 261-266.

[66] T.O. McBride, B.W. Pogue, S. Jiang, U.L. Österberg and K.D. Paulsen, A parallel-detection frequency domain nearinfrared tomography system for hemoglobin imaging of the breast in vivo, Rev Sci Instrum 72 (2001), 1817-1824.

[67] D.A. Boas, T. Gaudette and S.R. Arridge, Simultaneous imaging and optode calibration with diffuse optical tomography, Opt Express 8(5) (2001), 263-270.

[68] C.H. Schmitz, M. Löcker, J.M. Lasker, A.H. Hielscher and R.L. Barbour, Instrumentation for fast functional optical tomography, Rev Sci Instrum 73(2) (2002), 429-439.

[69] A. Ishimaru, Diffusion of light in turbid material, Appl Opt 28(12) (1989), 2210-2215.

[70] A. Ishimaru, Wave propagation and scattering in random media, Academic, New York, 1978.

[71] A.H. Hielscher, R.E. Alcouffe and R.L. Barbour, Comparison of finite-difference transport and diffusion calculations for photon migration in homogeneous and heterogeneous tissue, Phys Med Biol 43 (1998), 1285-1302.

[72] H. Dehghani, D.T. Delpy and S.R. Arridge, Photon migration in non-scattering tissue and the effects on image reconstruction, Phys Med Biol 44(12) (1999), 2897-2906.

[73] J. Ripoll, M. Nieto-Vesperinas, S.R. Arridge and H. Dehghani, Boundary conditions for light propagation in diffusive media with nonscattering regions, J Opt Soc Am A 17(9) (2000), 1671-1682.

[74] S.R. Arridge, H. Dehghani, M. Schweiger and E. Okada, The finite element model for the propagation of light in scattering media: a direct method for domains with nonscattering regions, Med Phys 27(1) (2000), 252-265.

[75] M. Firbank, S.R. Arridge, M. Schweiger and D.T. Delpy, An investigation of light transport through scattering bodies with non-scattering regions, Phys Med Biol 41(4) (1996), 767.

[76] E. Okada, M. Firbank, M. Schweiger, S.R. Arridge, M. Cope and D.T. Delpy, Theoretical and experimental investigation of near-infrared light propagation in a model of the adult head, Appl Opt 36(1) (1997), 21-32.

[77] M. Schweiger and S.R. Arridge, Optical tomographic reconstruction in a complex head model using a priori region boundary information, Phys Med Biol 44(11) (1999), 2703.

[78] S.R. Hintz, W.F. Cheong, J.P. van Houten, D.K. Stevenson and D.A. Benaron, Bedside imaging of intracranial hemorrhage in the neonate using light: comparison with ultrasound, computed tomography, and magnetic resonance imaging, $\mathrm{Pe}$ diatr Res 45 (1999), 54-59. 
[79] J.P. Vanhouten, D.A. Benaron, S. Spilman and D.K. Stevenson, Imaging brain injury using time-resolved near-infrared light scanning, Pediatr Res 39 (1996), 470-476.

[80] S.P. Gopinath, C.S. Robertson, C.F. Contant, R.K. Narayan, R.G. Grossman and B. Chance, Early detection of delayed traumatic intracranial hematomas using near-infrared spectroscopy, J Neurosurg 83 (1995), 438-444.

[81] S.P. Gophinath, C.S. Robertson, R.G. Grossman and B. Chance, Near-infrared spectroscopic localization of intracranial hematomas, J Neurosurg 79(1) (1993), 43-47.

[82] Y. Hoshi, I. Oda, Y. Wada, Y. Ito, Y. Yamashita, M. Oda, K. Ohta, Y. Yamada and M. Tamura, Visuospatial imagery is a fruitful strategy for the digit span backward task: a study with near-infrared optical tomography, Brain Res Cogn Brain Res 9 (2000), 339-342.

[83] B. Chance, E. Anday, S. Nioka, S. Zhou, L. Hong, K. Worden, C. Li, T. Murray, Y. Ovetsky, D. Pidikiti and R. Thomas, A novel method for fast imaging of brain function, noninvasively, with light, Opt Express 2(10) (1998), 411-423.

[84] D.A. Benaron, S.R. Hintz, A. Villringer, D. Boas, A. Kleinschmidt, J. Frahm, C. Hirth, H. Obrig, J.C. Van Houten, E.L. Kermit, W. Cheong and D.K. Stevenson, Noninvasive functional imaging of human brain using light, J Cereb Blood Flow Metab 20 (2000), 469-477.

[85] H. Obrig, R. Wenzel, M. Kohl, S. Horst, P. Wobst, J. Steinbrink, F. Thomas and A. Villringer, Near-infrared spectroscopy: does it function in functional activation studies of the adult brain? Int J Psychophysiol 35 (2000), 125-142.

[86] A. Villringer and B. Chance, Non-invasive optical spectroscopy and imaging of human brain function, Trends Neurosci 20 (1997), 435-442.

[87] A. Kleinschmidt, H. Obrig, M. Requardt, K. Merboldt, U. Dirnagl, A. Villringer and J. Frahm, Simultaneous recording of cerebral blood oxygenation changes during human brain activation by magnetic resonance imaging and near infrared spectroscopy, J Cereb Blood Flow Metab 16(5) (1996), 817826.

[88] G. Gratton and M. Fabiani, Dynamic brain imaging: eventrelated optical signal (EROS) measures of the time course and localization of cognitive-related activity, Psychon Bull Rev 5 (1995), 535-563.

[89] X.F. Cheng and D.A. Boas, Systematic diffuse optical image errors resulting from uncertainty in the background optical properties, Opt Express 4 (1999), 299-307, http://www.opticsexpress.org/oearchive/source/9108.htm.

[90] S. Wray, M. Cope and C.T. Delpy, Characteristics of the near infrared absorption spectra of cytochrome aa3 and hemoglobin for the noninvasive monitoring of cerebral oxygenation, Biochim Biophys Acta 933 (1988), 184-192.

[91] G. Gratton, M.R. Gooman-Wood and M. Fabiani, Comparison of Neural and Hemodynamic Measures of the Brain Response to Visual Stimulation: an Optical Imaging Study, Hum Brain Mapp 13 (2001), 13-25.

[92] G. Gratton, M. Fabiani, P.M. Corballis, D.C. Hood, M.R. Goodman-Wood, J. Hirsch, K. Kim, D. Friedman and E. Gratton, Fast and localized event-related optical signals (EROS) in the human occipital cortex: comparisons with the visual evoked potential and fMRI, Neuroimage 6 (1997), $168-180$.

[93] J. Steinbrink, M. Kohl, H. Obrig, G. Curio, F. Syré, F. Thomas, H. Wabnitz, H. Rinneberg and A. Villringer, Somatosensory evoked fast optical intensity changes detected non-invasively in the adult human head, Neurosci Lett 291 (2000), 105-108.
[94] U. Wolf, M. Wolf, V. Toranov, A. Michalos, L.A. Paunescu and E. Gratton, Detecting cerebral functional slow and fast signals by frequency-domain near-infrared spectrocopy using two different sensors, in Biomedical Topical Meetings, OSA Technical Digest, Optical Society of America, Washington, DC, 2000, pp. 427-429.

[95] M.A. Franceschini, J. Thompson, J.P. Culver, G. Strangman and D.A. Boas, Looking for the fast signal: neuronal and hemodynamic evoked responses of the sensory-motor cortex, in Technical Digest of the OSA Biomedical Topical Meeting on Advances in Optical Imaging and Photon Migration Optical Society of America, Washington, DC, 2002, pp. 208-210.

[96] D.G. Hirtz, Report of the National Institute of Neurological Disorder and Stroke workshop on near infrared spectroscopy, Pediatrics 91(2) (1993), 414-417.

[97] S.R. Hintz, W-F Cheong, J.P. van Houten, D.K. Stevenson and D.A. Benaron, Bedside imaging of intracranial hemorrhage in the neonate using light: comparison with ultrasound, computed tomography, and magnetic resonance imaging, $P e$ diatrics 45(1) (1999), 54-59.

[98] R.A. Kline, W. Negendank, L. McCoy and R. Berguer, Benificial effects of isovolemic hemodilution using a perfluorocarbon emulsion in a stroke model, Am J Surg 162 (1991), $103-106$.

[99] R.A. Kline, W.G. Negendank, L.E. McCoy, M. Lester and R. Berguer, MRI quantification of edema in focal cerebral ischemia in cats: correlation with cytochrome aa3 oxidation state, Magnet Reson Med 13 (1990), 319-323.

[100] F.F. Jöbsis, J.H. Keizer, J.C. LaManna and M. Rosenthal, Reflectance spectrophotometry of cytochrome aa3 in vivo, $J$ Appl Physiol 43 (1977), 858-872.

[101] F.F. Jöbsis, Noninvasive, near-infared monitoring of cellular oxygen sufficiency in vivo, Adv Exp Med Biol 191 (1985), 833-842.

[102] H. Miyake, S. Nioka, A. Zaman, D.S. Smith and B. Chance, The detection of cytochrome oxidase heme iron and copper absorption in the blood-perfused and blood-free brain in normoxia and hyoxia, Anal Biochem 192 (1991), 149-155.

[103] C.E. Cooper, M. Cope, R. Springett, P.N. Amess, J. Penrice, L. Tyszczuk, S. Punwani, R. Ordidge, J. Wyatt and D.T. Delpy, Use of mitochondrial inhibitors to demonstrate that cytochrome oxidase near-infared spectroscopy can measure mitochondrial dysfunction noninvasively in the brain, $J$ Cereb Blood Flow Metab 19(61) (1999), 27-38.

[104] K. Uludag, M. Kohl, J. Steinbrink, H. Obrig and A. Villringer, Cross talk in the Lambert-Beer calculation for nearinfrared wavelengths estimated by Monte Carlo simulations, J Biomed Opt 7(1) (2002), 51-59.

[105] T. Wolf, U. Lindauer, H. Obrig, J. Dreier, T. Back, A. Villringer and U. Dirnagl, Systemic nitric oxide synthase inhibition does not affect brain oxygenation during cortical spreading depression in rats: a noninvasive near-infrared spectroscopy and laser-doppler flowmetry study, J Cereb Blood Flow Metab 16(6) (1996), 1100-1107.

[106] M. Lauritzen, Regional cerebral blood flow during cortical spreading depression in rat brain: increased reactive hyperfusion in low-flow states, Acta Neurol Scand 75 (1987), 1-8.

[107] R.B. Duckrow, Regional cerebral blood flow during spreading cortical depression in conscious rats, J Cereb Blood Flow Metab 11 (1991), 150-154.

[108] R.B. Duckrow, A brief hypoperfusion precedes spreading depression if nitric oxide synthesis is inhibited, Brain Res 618 (1993), 190-195. 
[109] T. Wolf , U. Lindauer, U. Reuter, T. Back, A. Villringer, K. Einhäupl and U. Dirnagl, Noninvasive near infrared spectroscopy monitoring of regional cerebral blood oxygenation changes during peri-infarct depolarization in focal cerebral ischemia in the rat, J Cereb Blood Flow Metab 17 (1997), 950-954.

[110] F. Vernieri, N. Rosato, F. Pauri, F. Tibuzzi, F. Passarelli and P.M. Rossini, Near infared spectroscopy and transcranial doppler in monohemispheric stroke, Eur Neurol 41 (1999), 159-162.

[111] E.M. Nemoto, H. Yonas and A. Kassam, Clinical experience wih cerebral oximetry in stroke and cardiac arrest, Crit Care Med 28(4) (2000), 1052-1054.

[112] W. Chen, P.C. Li, Q.M. Luo, S.Q. Zeng and B. Hu, Hemodynamic assessment of ischemic stroke with near-infrared spectroscopy, Space Med Med Eng 13(2) (2000), 84-89.

[113] W. Chen, G. Lu and W. Lichty, Localizing the focus of ischemic stroke with near infrared spectroscopy, Chin Med J 115(1) (2002), 84-88.

[114] A.Y. Bluestone, G. Abdoulaev, C. Schmitz, R.L. Barbour and A.H. Hielscher, Three-dimensional optical-tomography of hemodynamics in the human head, Opt Express 9(6) (2001), 272-286. (http://www.opticsexpress.org/abstract.cfm?URI= OPEX-9-6-272)

[115] F.P. Tiecks, C. Douville, S. Byrd, A.M. Lam and D.W. Newell, Evaluation of impaired cerebral autoregulation by the Valsalva Maneuver, Stroke 27 (1996), 1177-1182.

[116] B.F. Matta, J. Lam and P. Smileswiski, The effect of the Valsalva maneuver on cerebral hemodynamics: a near infrared spectroscopy study, J Neurosurg Anesthesiol 8 (1996), 601.

[117] S.L. Dawson, R.B. Panerai and J.F. Potter, Critical closing pressure explains cerebral hemodynamics during the Valsalva maneuver, J Appl Physiol 86 (1999), 675-680.

[118] Y. Pei, H.L. Graber and R.L. Barbour, Influence of systematic errors in reference states on image quality and on stability of derived information for dc optical imaging, Appl Opt 40(31) (2001), 5755-5769.

[119] E.M.C. Hillman, H. Dehghani, J.C. Hebden, S.R. Arridge, M. Schweiger and D.T. Delpy, Differential imaging in heterogeneous media: limitations of linearization assumptions in optical tomogrphy, in: Optical Tomography and Spectroscopy of Tissue IV, B. Chance, R.R. Alfano, B.J. Tromberg, M. Tamura, and E.M. Sevick-Muraca, eds, Proc. SPIE Vol/. 4250, 2001, pp. 327-338.

[120] R. Weissleder, C.H. Tung, U. Mahmood and A. Bogdanov Jr., In vivo imaging of tumors with protease activated nearinfra-red fluorescent probes, Nat Biotechnol 17 (1999), 375378.

[121] U. Mahmood, C.H. Tung, A. Bogdanov Jr., and R. Weissleder, Near-infrared optical imaging of protease activity for tumor detection, Radiology 213 (1999), 866-870.

[122] T.J. Sweeney, V. Mailander, A.A. Tucker et al., Visualizing the kinetics of tumor-cell clearance in living animals, Proc Natl Acad Sci USA 96 (1999), 12044-12049.

[123] C.H. Contag, D. Jenkins, P.R. Contag and R.S. Negrin, Use of reporter genes for optical measurements of neoplastic disease in vivo, Neoplasia 2 (2000), 41-52.

[124] C.H. Contag, S.D. Spilman, P.R. Contag et al., Visualizing gene expression in living mammals using a bioluminescent reporter, Photochem Photobiol 66 (1997), 523-531.

[125] V. Ntziachristos and R. Weissleder, Experimental threedimensional fluorescence reconstruction of diffuse media by use of a normalized born approximation, Opt Lett 26(12) (2001), 893-895.
[126] M. Nemoto, Y. Nomura, C. Sato, M. Tamura, K. Houkin, I. Koyanagi and H. Abe, Analysis of optical signals evoked by peripheral nerve stimulation in rat somatosensory cortex: dynamic changes in hemoglobin concentration and oxygenation, J Cereb Blood Flow Metab 19(3) (1999), 246-259.

[127] C. Cheung, J.P. Culver, K. Takahashi, J.H. Greenberg and A.G. Yodh, In vivo cerebrovascular measurement combining diffuse near-infrared absorption and correlation spectroscopies, Phys Med Biol 46(8) (2001), 2053-2065.

[128] B.W. Pogue and K.D. Paulsen, High-resolution near-infrared tomographic imaging simulations of the rat cranium by use of a priori magnetic resonance imaging structural information, Opt Lett 23(21) (1998), 1716-1718.

[129] J. Norris, Senior Editor, Professional guide to diseases, 5th Edition, Springhouse Corp, Springhouse, PA, 1998, pp. 355360.

[130] E.D. Harris Jr., Rheumatoid arthritis: pathophysiology and implications for therapy, N Engl J Med 322 (1990), 12771289.

[131] D.P. Lubeck, The economic impact of arthritis, Arthritis Care Res 8 (1995), 304-310.

[132] P.F. Laudicina, Applied Pathology for Radiographers, Philadelphia, PA: W.B. Saunders Company, 1989, pp. 147.

[133] O. Steinbrocker, C.H. Traeger and R.C. Batermann, Therapeutic criteria in rheumatoid arthritis, J Amer Med Ass 140(8) (1949), 659-xx.

[134] J. Beuthan, V. Prapavat, R.D. Naber, O. Minet and G. Müller, Diagnosis of inflammatory rheumatic diseases with photon density waves, Proc. 2676 SPIE-The International Society for Optical Engineering, Biomedical Sensing, Imaging, and Tracking Technologies I, R.A. Lieberman, H. Podbielska and T. Vo-Dinh, eds, 1996, pp. 43-53.

[135] A. Klose, V. Prapavat, O. Minet, J. Beuthan and G. Mueller, RA diagnostics applying optical tomography in frequencydomain, Proc. 3196 SPIE-The International Society for Optical Engineering, 1997, pp. 194-204.

[136] A.D. Klose, A.H. Hielscher, K.M. Hanson and J. Beuthan, Three-dimensional optical tomography of a finger joint model for diagnostic of rheumatoid arthritis, Proc. 3566 SPIE-The International Society for Optical Engineering, 1998, pp. 151160 .

[137] U. Netz, J. Beuthan, H.J. Capius, H.C. Koch, A.D. Klose and A.H. Hielscher, Imaging of rheumatoid arthritis in finger joints by sagittal optical tomography, Medical Laser Application 16 (2001), 306-310.

[138] Y. Xu, N. Iftimia, H.B. Jiang, L.L. Key and M.B. Bolster, Three-dimensional diffuse optical tomography of bones and joints, J Biomed Opt 7(1) (2002), 88-92.

[139] Y. Xu, N. Iftimia, H.B. Jiang, L.L. Key and M.B. Bolster, Imaging of in vitro and in vivo bones and joints with continuous-wave diffuse optical tomography, Opt Express 8(7) (2001), 447-451.

[140] J.M. Lasker, A. Bluestone, C. Schmitz, R.L. Barbour and A.H. Hielscher, Volumetric optical-tomographic dynamic imaging of small tissue volumes, in Technical Digest of Biomedical Topical Meeting on Advances in Optical Imaging and Photon Migration Optical Society of America, Washington, DC, 2002, pp. 524-526.

[141] H. Jess, H. Erdl, K.T. Moesta, S. Fantini, M.A. Franceschini, E. Gratton and M. Kaschke, Intensity modulated breast imaging: technology and clinical pilot study results, in: $O S A$ Trends in Optics and Photonics on Advances in Optical Imaging and Photon Migration, Vol. 2, R.R. Alfano and J.G. Fu- 
jimoto, eds, Optical Society of America, Washington, DC, 1996, pp. 126-129.

[142] O. Jarlman, R. Berg, S. Andersson-Engels, S. Svanberg and H. Pettersson, Time-resolved white light transillumination for optical imaging, Acta Radiologica 38 (1997), 185-189.

[143] S. Nioka, Y. Yung, M. Shnall, S. Zhao, S. Orel, C. Xie, B. Chance and L. Solin, Optical imaging of breast tumor by means of continuous waves, Adv Exp Med Biol 411 (1997), 227-232.

[144] B.J. Tromberg, O. Coquoz, J.B. Fishkin, T. Pham, E.R. Anderson, J. Butler, M. Cahn, J.D. Gross, V. Venugopalan and D. Pham, Non-invasive measurements of breast tissue optical properties using frequency-domain photon migration, Philos Trans R Soc Lond B Biol Sci 352 (1997), 661-668.

[145] R.R. Alfano, S.G. Demos and S.K. Gayen, Advances in optical imaging of biomedical media, Ann NY Acad Sci $\mathbf{8 2 0}$ (1997), 248-70; Discussion 271.

[146] S. Fantini, M.A. Franceschini, G. Gaida, E. Gratton, H. Jess, W.W. Mantulin, K.T. Moesta, P.M. Schlag and M. Kaschke,
Frequency-domain optical mammography: edge effect corrections, Med Phys 23 (1996), 149-157.

[147] V. Ntziachristos, A.G. Yodh, M.D. Schnall and B. Chance, MRI-guided diffuse optical spectroscopy of malignant and benign breast lesions, Neoplasia 4(4) (2002), 347-354 .

[148] P.R. Contag, Whole-animal cellular and molecular imaging to accelerate drug development, Drug Discov Today 7(10) (2002), 555-562.

[149] R. Weissleder and U. Mahmood, Molecular imaging, Radiology 219 (2001), 316-333.

[150] D.Y. Paithankar, A.U. Chen, B.W. Pogue, M.S. Patterson and E.M. Sevick-Muraca, Imaging of fluorescent yield and lifetime from multiply scattered light reemitted from random media, Appl Opt 36 (1997), 2260-2272.

[151] R. Roy and E.M. Sevick-Muraca, Three-dimensional unconstrained and constrained image-reconstruction techniques applied to fluorescence, frequency-domain photon migration, Appl Opt 40 (2001), 2206-2215. 


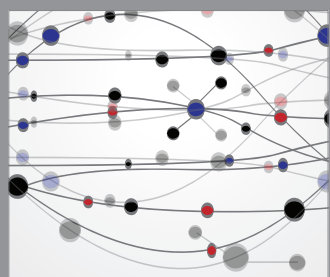

The Scientific World Journal
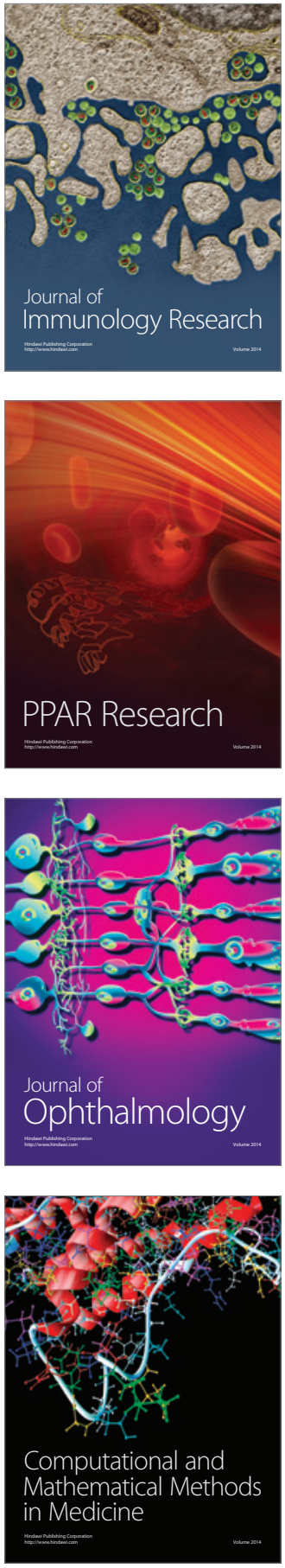

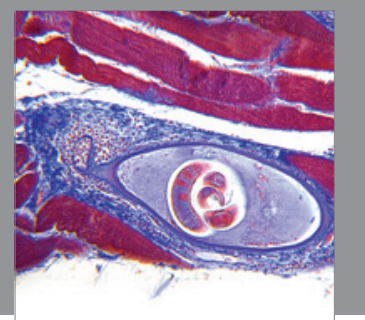

Gastroenterology

Research and Practice
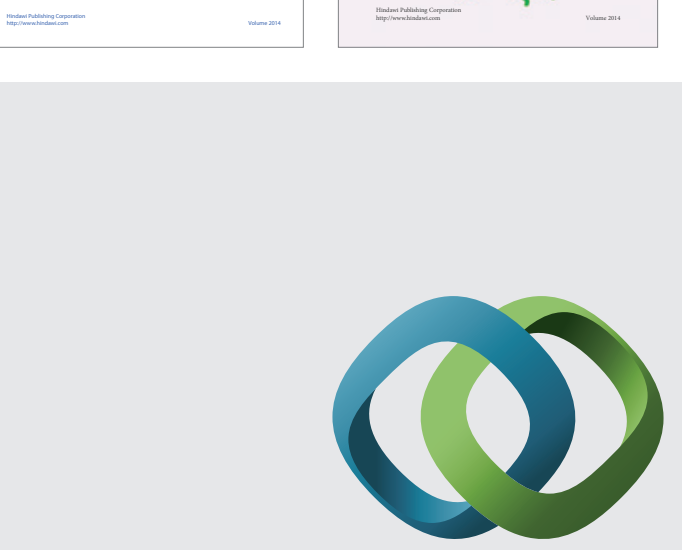

\section{Hindawi}

Submit your manuscripts at

http://www.hindawi.com
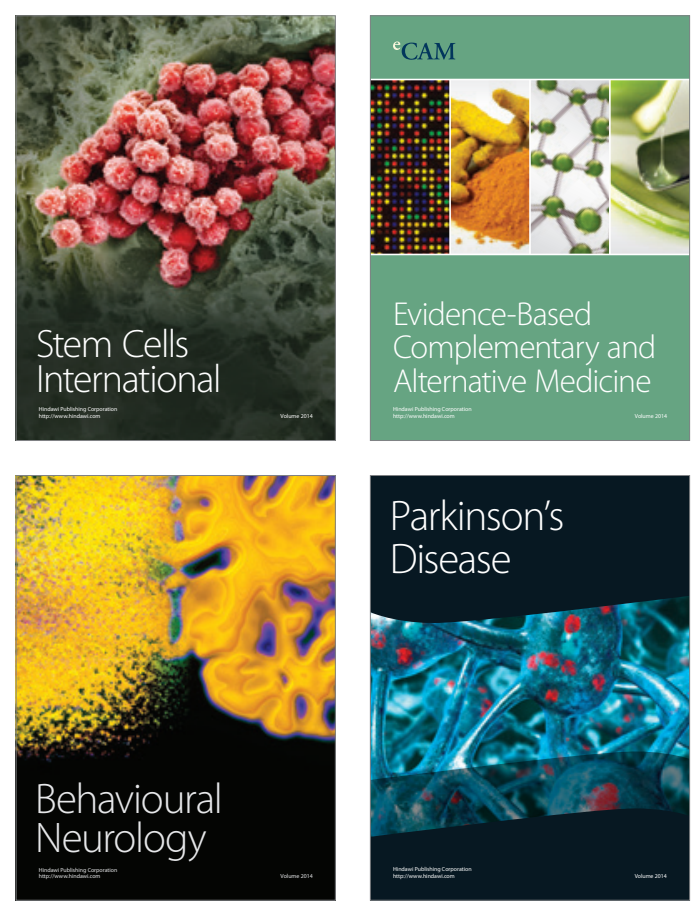

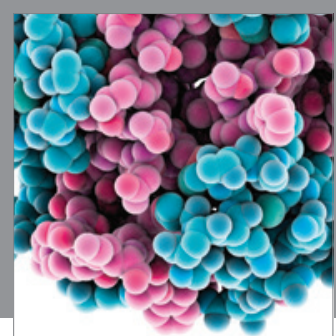

Journal of
Diabetes Research

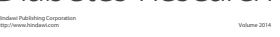

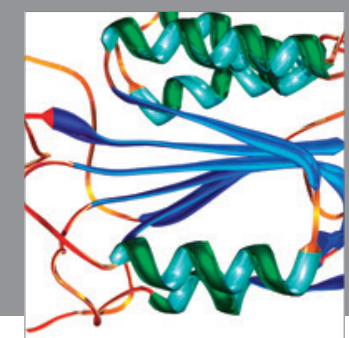

Disease Markers
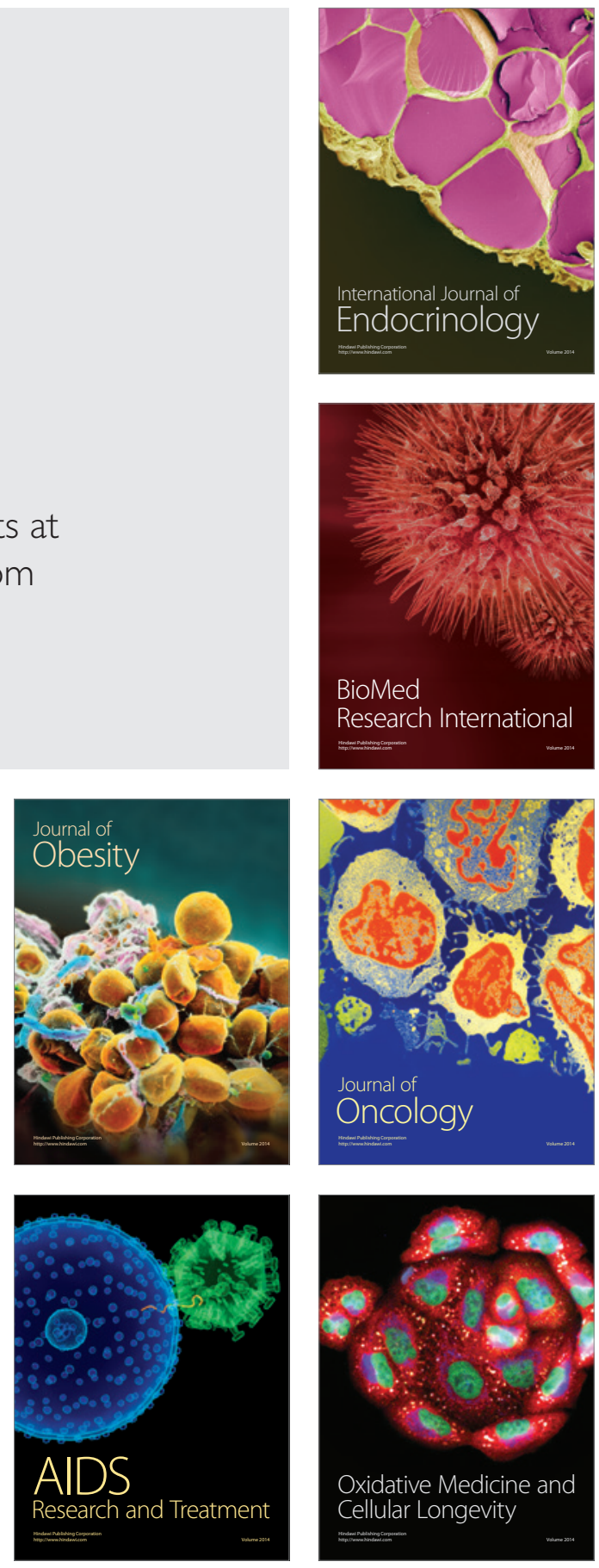